\title{
Global Regularity and Spectra of Laplace-Beltrami Operators on Pseudoconvex Domains
}

By

\author{
Kensho TAKEgOSHI*
}

\begin{tabular}{|c|c|}
\hline & Contents \\
\hline & Introduction $\ldots \ldots \ldots \ldots$ \\
\hline & Notations and basic facts.. \\
\hline & Statement of main results $\ldots \ldots \ldots \ldots$ \\
\hline & A priori estimates for smooth forms \\
\hline & Proof of main results $\ldots \ldots \ldots \ldots \ldots$ \\
\hline & Application to cohomology theory \\
\hline & Appendix $\quad \ldots \ldots \ldots \ldots \ldots \ldots$ \\
\hline
\end{tabular}

\section{$\S 1$. Introduction}

In the theory of elliptic differential operators, the result on Laplace-Beltrami operators defined on compact complex manifolds is a remarkable one and has many important applications to the cohomology theory on compact complex manifolds. On the other hand, in recent years, the property of Laplace-Beltrami operators on non-compact complex manifolds has been investigated from various aspects. In particular, the Kohn's solution to $\bar{\partial}$-Neumann problem is one of the most remarkable results (see [2] [6]). Looking back to our situation i. e. the cohomology theory on weakly 1-complete manifolds (for example, [10] [11] [13]), it seems that the Kohn's argument, which is based on $L^{2}$-estimates for the $\bar{\partial}$ operator, is applicable to the study of the cohomological property of weakly 1complete manifolds. In this paper, having this motivation in mind, and on the other hand, purely from the point of view of partial differential equations, we study the global boundary regularity and the behavior of spectra of LaplaceBeltrami operators on pseudoconvex domains. We apply the result to the cohomology theory of weakly 1-complete manifolds by showing an upper semi-continuity theorem for the dimension of the cohomology groups on a family of weakly 1complete manifolds. The plan of this paper is as follows. In Section 2, we prepare the notations needed in the latter sections and give a sufficient condition for the solvability of the $L^{2} \bar{\partial}$-Neumann problem. In Section 3, we state our main results. In Section 4, we show the basic estimate which is crucial to prove the regularization

Communicated by S. Nakano, May 11, 1982.

* Research Institute for Mathematical Sciences, Kyoto University, Kyoto 606, Japan. 
theorem $\mathrm{R}_{s, \mu}$. Our starting point to show it is the estimate (4.2) of Proposition 4.4 which is deduced from the formula (A.2.2) of Theorem A.2.1. We use this formula more effectively than the usage in our previous article [13] i. e. the term $\|\bar{\nabla} \varphi\|_{m}^{2}$ in (A.2.2) plays an important role to estimate the normal derivatives. In Section 5, using this basic estimate and the method of Kohn and Nirenberg in [6] [8], we prove our main results. In Section 6, combining this regularity result with the harmonic representation theorem of cohomology groups on weakly 1complete manifolds, we show an upper semi-continuity theorem. In Section 7, we give the proofs of Lemma 4.3 and Proposition 4.4 mentioned in Section 4 and refer to a fundamental fact on spectra of self-adjoint operators which we need.

The author expresses his hearty thanks to Dr. T. Ohsawa. Several useful discussions with him led the author to this problem. In particular, the upper semi-continuity theorem is the problem which originated from these discussions. He also expresses his gratitude to Professor S. Nakano, who is the proponent of the conception of weakly 1-complete manifolds, for his constant encouragement and kind advices. Last he expresses his thanks to the referee for valuable criticisms.

\section{$\S 2 . \quad$ Notations and Basic Facts}

Let $M$ be an $n$-dimensional complex manifold and let $E$ be a holomorphic line bundle on $M$. Let $E^{\otimes m}$ be the $m$-times tensor product of $E$ for positive integer $m$. For integers $p, q \geqq 0,0 \leqq s \leqq \infty, m \geqq 1$ and an open subset $Y \leqq M$, we define the following notations:

$C_{s}^{p, q}\left(Y, E^{\otimes m}\right)$ : the space of $E^{\otimes m}$-valued differential forms of type $(p, q)$ and of class $C^{s}$ on $Y$.

$C_{c, s}^{p, q}\left(Y, E^{\otimes m}\right)$ : the space of forms in $C_{s}^{p, q}\left(Y, E^{\otimes m}\right)$ with compact supports.

$C_{s}^{p, q}\left(\bar{Y}, E^{\otimes m}\right):$ the image of the restriction homomorphism from $C_{s}^{p, q}\left(M, E^{\otimes m}\right)$ to $C_{s}^{p, q}\left(Y, E^{\otimes m}\right)(Y \subsetneq M)$.

$C_{s}^{p, q}(Y) \quad$ : the space of differential forms of type $(p, q)$ and of class $C^{s}$ on $Y$.

$C_{c, s}^{p, q}(Y) \quad$ : the space of forms in $C_{s}^{p, q}(Y)$ with compact supports.

In particular, when $s=\infty$, we denote $C_{\infty}^{p, q}\left(Y, E^{\otimes m}\right)=C^{p, q}\left(Y, E^{\otimes m}\right), C_{c, \infty}^{p, q}\left(Y, E^{\otimes m}\right)=$ $C_{c}^{p, q}\left(Y, E^{\otimes m}\right)$ etc. for simplicity. Let $\left\{e_{i j}\right\}$ be a system of transition functions of $E$ with respect to a covering $\left\{U_{i}\right\}_{i \in I}$. We express $\varphi=\left\{\varphi_{i}\right\} \in C_{s}^{p, q}\left(M, E^{\otimes m}\right)$ as $\varphi_{i}=1 / p ! q ! \sum_{c_{1} \cdots c_{p}, d_{1} \cdots d_{q}} \varphi_{i} c_{1} \cdots c_{p}, \bar{a}_{1} \cdots \bar{d}_{q} d z_{\imath}^{c_{1}} \wedge \cdots \wedge d z_{\imath}^{c} p \wedge d z_{\imath}^{\bar{d}_{1}} \wedge \cdots \wedge d z_{i}^{\bar{d} q}$. For simplicity, we sometimes write $\varphi_{i}=1 / p ! q ! \sum_{C_{p}, D_{q}} \varphi_{i, C_{p}, \bar{D}_{q}} d z_{\imath}^{C} p \wedge d z_{\imath}^{\bar{D}_{q}}$ where $C_{p}=\left(c_{1}, \cdots, c_{p}\right)$, $D_{q}=\left(d_{1}, \cdots, d_{q}\right)$ and so on. Let

$$
d s^{2}=\sum_{\alpha, \beta=1}^{n} g_{i, \alpha \bar{\beta}} d z_{\imath}^{\alpha} d z_{\imath}^{\bar{\beta}}
$$

be a hermitian metric on $M$. Let

$$
\boldsymbol{a}=\left\{a_{i}\right\}
$$


be a hermitian metric of $E=\left\{e_{i j}\right\}$ with respect to the covering $\left\{U_{i}\right\}_{i \in I}$ i.e. $\boldsymbol{a}=$ $\left\{a_{i}\right\}$ satisfies $a_{i}\left|e_{i j}\right|^{2}=a_{j}$ on $U_{i} \cap U_{j}$. Here we assume that these metrics are of $C^{\infty}$ class. We set

and

$$
a_{\imath}^{m} \varphi_{i} \wedge * \bar{\psi}_{i}=\langle\varphi, \phi\rangle_{m} d V \quad \text { for } \varphi, \phi \in C_{s}^{p, q}\left(M, E^{\otimes m}\right)
$$

$$
\chi \wedge * \bar{\omega}=\langle\chi, \omega\rangle d V \quad \text { for } \chi, \omega \in C_{s}^{p, q}(M)
$$

where $*$ is the star operator and $d V$ is the volume element with respect to $d s^{2}$.

For an open subset $Y \subseteq M$, we define

$$
(\varphi, \psi)_{m, Y}=\int_{Y}\langle\varphi, \psi\rangle_{m} d V \quad \text { if } \varphi \text { or } \psi \in C_{c, s}^{p, q}\left(Y, E^{\otimes m}\right)
$$

and

$$
(\chi, \omega)_{Y}=\int_{Y}\langle\chi, \omega\rangle d V \quad \text { if } \chi \text { or } \omega \in C_{c, s}^{p, q}(Y)
$$

We set

$$
\begin{array}{cl}
\|\varphi\|_{m, Y}^{2}=(\varphi, \varphi)_{m, Y} & \text { for } \varphi \in C_{c, s}^{p, q}\left(Y, E^{\otimes m}\right) \\
\|\chi\|_{Y}^{2}=(\chi, \chi)_{Y} & \text { for } \chi \in C_{c, s}^{p, q}(Y)
\end{array}
$$

respectively.

From now on, let $X$ be a relatively compact domain on $M$ with smooth boundary $\partial X$ i. e. there exist a neighborhood $\Omega$ of $\partial X$ and a real-valued $C^{\infty}$ function $h$ on $\Omega$ such that $\Omega \cap X=\{x \in \Omega \mid h(x)<0\}$ and the gradient of $h$ nowhere vanishes on $\partial X$. For each element $U_{i}$ of $\left\{U_{i}\right\}_{i \in I}$, let $\left(z_{i}^{1}, \cdots, z_{\imath}^{n}\right)$ be local coordinates on $U_{i}$. We separate $z_{i}^{k}$ into the real and imaginary parts: $z_{i}^{k}=$ $x_{i}^{2 k-1}+\sqrt{-1} x_{i}^{2 k}(k=1,2, \cdots, n)$. For any multi-index $\sigma=\left(\sigma_{1}, \cdots, \sigma_{2 n}\right)$, we set $D_{i}^{\sigma}=(-\sqrt{-1})^{\mid \sigma^{\prime}}\left(\partial / \partial x_{i}^{1}\right)^{\sigma_{1}} \cdots\left(\partial / \partial x_{i}^{2 n}\right)^{\sigma_{2 n}}$ where each $\sigma_{i}$ is a non-negative integer and $|\sigma|=\sum_{k=1}^{2 n} \sigma_{k}$. We take a family $\left\{\rho_{i}\right\}_{i \in I}$ of $C^{\infty}$-functions on $M$ such that i) supp $\rho_{i} \Subset U_{i}$ ii) $0 \leqq \rho_{i} \leqq 1$ if $U_{i} \cap \bar{X} \neq \varnothing$ (we may assume that such $i$ are finitely many), $\rho_{i}=0$ if $U_{i} \cap \bar{X}=\varnothing$ and iii) $\sum_{i \in I} \rho_{i} \equiv 1$ on $\bar{X}$. For $\varphi \in C^{p, q}\left(\bar{X}, E^{\otimes m}\right)$ and a multi-index $\sigma=\left(\sigma_{1}, \cdots, \sigma_{2 n}\right)$, we set

$$
D_{\imath}^{\sigma}\left(\rho_{i} \varphi_{i}\right)=\frac{1}{p ! q !} \sum_{p, D_{q}} D_{\imath}^{\sigma}\left(\rho_{i} \varphi_{i, C_{p}, \bar{D}_{q}}\right) d z_{\imath}^{C} p \wedge d z_{\imath}^{\bar{D}_{q}} \quad \text { for } i \in I .
$$

For every non-negative integer $s$, we define the norm \|\|$_{s, X}^{2}$ on $C^{p, q}\left(\bar{X}, E^{\otimes m}\right)$ by

for $\varphi=\left\{\varphi_{i}\right\} \in C^{p, q}\left(\bar{X}, E^{\otimes m}\right)$.

$$
\|\varphi\|_{s, X}^{2}=\sum_{\substack{0 \leq 1 \sigma 1 \leq s \\ i \in I}}\left\|D_{i}^{\sigma}\left(\rho_{i} \varphi_{i}\right)\right\|_{X}^{2}
$$

In particular, we set $\|\varphi\|_{X}=\|\varphi\|_{0, X}$.

Remark 2.7. The norm \|\|$_{s, x}$ is independent of the choice of coverings and their local coordinates up to equivalence. 
From now on, in the above notations, the presentation of domains of integration will be omitted when they are clearly understood.

For integers $p, q \geqq 0, m \geqq 1$ and $s \geqq 0$, we define the following spaces:

$L^{p, q}\left(X, E^{\otimes m}\right)$ : the Hilbert space obtained by completing $C_{c}^{p, q}\left(X, E^{\otimes m}\right)$ under the norm \|\|$_{m, x}^{2}$.

$\mathcal{C}_{s}^{p, q}\left(\bar{X}, E^{\otimes m}\right):$ the Hilbert space obtained by completing $C^{p, q}\left(\bar{X}, E^{\otimes m}\right)$ under the norm \|\|$_{s, X}^{2}$.

Remark 2.8. By Remark 2.7, $\mathcal{C}_{s}^{p, q}\left(\bar{X}, E^{\otimes m}\right)$ is well defined as a topological vector space. In particular, $\mathcal{C}_{v}^{p, q}\left(\bar{X}, E^{\otimes m}\right)$ coincides with $L^{p, q}\left(X, E^{\otimes m}\right)$ as topological vector spaces.

Moreover if $t>s \geqq 0$, then there is a natural embedding $:: \mathcal{C}_{t}^{p, q}\left(\bar{X}, E^{\otimes m}\right) \hookrightarrow$ $\mathcal{C}_{s}^{p, q}\left(\bar{X}, E^{\otimes m}\right)$. With respect to the spaces $\mathcal{C}_{s}^{p, q}\left(\bar{X}, E^{\otimes m}\right)$, the Rellich and Sobolev lemmas hold (see [2] p. 124 (A.2.3) Proposition).

Lemma 2.1. 1) If $t>s \geqq 0$ are integers, then the inclusion $\iota: \mathcal{C}_{t}^{p, q}\left(\bar{X}, E^{\otimes m}\right)$ $C_{\rightarrow} \mathcal{C}_{s}^{p, q}\left(\bar{X}, E^{\otimes m}\right)$ is compact.

2) If $s \geqq n+1$, then $\mathcal{C}_{s}^{p, q}\left(\bar{X}, E^{\otimes m}\right) \hookrightarrow_{C} C_{s-n-1}^{p, q}\left(\bar{X}, E^{\otimes m}\right) \quad\left(n=\operatorname{dim}_{C} M\right)$.

We have the operator $\bar{\partial}: C^{p, q}\left(X, E^{\otimes m}\right) \rightarrow C^{p, q+1}\left(X, E^{\otimes m}\right)$ and denote by $\vartheta_{m}$ the formal adjoint operator of $\bar{\partial}$ in $C_{\vec{c} \cdot}^{\cdot}\left(X, E^{\otimes m}\right)$ with respect to $(,)_{m, X}$. We denote again by $\bar{\partial}$ the operator from $L^{p, q}\left(X, E^{\otimes m}\right)$ to $L^{p, q+1}\left(X, E^{\otimes m}\right)$ extending the original $\bar{\partial}$ : thus a form $\varphi \in L^{p, q}\left(X, E^{\otimes m}\right)$ is in the domain of $\bar{\partial}$ if and only if $\bar{\partial} \varphi$, defined in the sense of distribution, belongs to $L^{p, q+1}\left(X, E^{\otimes m}\right)$. Then $\bar{\partial}$ is a densely defined closed operator. Hence the adjoint operator $\bar{\partial}_{m}^{*}$ of $\bar{\partial}$ in $L^{\cdot \cdot \cdot}\left(X, E^{\otimes m}\right)$ can be defined. In general, given Hilbert spaces $H_{1}$ and $H_{2}$, and a densely defined closed operator $T: H_{1} \rightarrow H_{2}$, we denote by $T^{*}$ its adjoint operator from $H_{2}$ to $H_{1}$ and denote their domains, ranges and nullities by $D_{T}, D_{T^{*}}, R_{T}$ $R_{T^{*}}, \quad N_{T}$ and $N_{T^{*}}$ respectively. In the case when $H_{1}=L^{p, q}\left(X, E^{\otimes m}\right), H_{2}=$ $L^{p, q+1}\left(X, E^{\otimes m}\right)$ and $T=\overline{\bar{o}}$, we let $D_{\bar{\partial}}=D_{\bar{\partial}}^{p, q}, R_{\bar{\partial}}=R_{\bar{\partial}}^{p, q+1}$ and $N_{\bar{o}}=N_{\bar{\partial}}^{p}, q$ and so on.

Let $d S$ be the volume element of the real differentiable manifold $\partial X$, defined by the equation $d V=d h /|\operatorname{grad} h|_{d s 2} \wedge d S$ on $\partial X$, where $h$ is the defining function of $X$. Then by integration by parts, we have

$$
(\bar{\partial} \varphi, \psi)_{m}=\left(\varphi, \vartheta_{m} \psi\right)_{m}+(-1)^{p} \int_{\partial X}\langle\varphi, *(\partial h \wedge * \psi)\rangle d S
$$

for $\varphi \in C_{s}^{p, q}\left(\bar{X}, E^{\otimes m}\right)$ and $\phi \in C_{s}^{p, q+1}\left(\bar{X}, E^{\otimes m}\right)(1 \leqq s \leqq \infty)$.

We define the subspace $B_{s}^{p, q}\left(\bar{X}, E^{\otimes m}\right)$ of $C_{s}^{p, q}\left(\bar{X}, E^{\otimes m}\right)$ by

$$
B_{s}^{p, q}\left(\bar{X}, E^{\otimes m}\right)=\left\{\varphi \in C_{s}^{p, q}\left(\bar{X}, E^{\otimes m}\right) \mid \partial h \wedge * \varphi=0 \text { on } \partial X\right\}
$$

for every $1 \leqq s \leqq \infty$.

In particular, we denote $B_{\infty}^{p, q}\left(\bar{X}, E^{\otimes m}\right)=B^{p, q}\left(\bar{X}, E^{\otimes m}\right)$ for simplicity. From (2.9), we have 


$$
(\bar{\partial} \varphi, \phi)_{m}=\left(\varphi, \vartheta_{m} \psi\right)_{m}
$$

whenever $\varphi \in C_{s}^{p, q}\left(\bar{X}, E^{\otimes m}\right)$ and $\phi \in B_{s}^{p, q+1}\left(\bar{X}, E^{\otimes m}\right)(1 \leqq s \leqq \infty)$. On $B^{p, q}\left(\bar{X}, E^{\otimes m}\right)$ we define the hermitian forms $D_{m}$ and $Q_{m}: B^{p, q}\left(\bar{X}, E^{\otimes m}\right) \times B^{p, q}\left(\bar{X}, E^{\otimes m}\right) \rightarrow C$ by

and

$$
D_{m}(\varphi, \phi)=(\bar{\partial} \varphi, \vec{\partial} \psi)_{m}+\left(\vartheta_{m} \varphi, \vartheta_{m} \psi\right)_{m}
$$

$$
Q_{m}(\varphi, \phi)=D_{m}(\varphi, \phi)+(\varphi, \phi)_{m} .
$$

Let $W^{p, q}\left(X, E^{\otimes m}\right)$ be the Hilbert space obtained by completing $B^{p, q}\left(\bar{X}, E^{\otimes m}\right)$ under the norm $Q_{m}(,)^{1 / 2}$. Since $\|\varphi\|_{m}^{2} \leqq Q_{m}(\varphi, \varphi)$ for $\varphi \in W^{p, q}\left(X, E^{\otimes m}\right), W^{p, q}\left(X, E^{\otimes m}\right)$ can be considered as a subspace of $L^{p, q}\left(X, E^{\otimes m}\right)$.

We recall the following well known theorem (see [15] Theorem 5.36).

Theorem 2.2. Let $(H,()$,$) be a Hilbert space and let H_{1}$ be a dense subspace of $H$. Assume that a hermitian inner product (, $)_{1}$ is defined on $H_{1}$ in such a way that $\left(H_{1},(,)_{1}\right)$ is a Hilbert space and with some positive constant $C$ we have $\|f\|^{2} \leqq C\|f\|_{1}^{2}$ for all $f \in H_{1}$. Then there exists exactly one self-adjoint operator $F$ (i.e. $F$ is densely defined and $F=F^{*}$ ) on $H$, which is called the Friedrichs operator associated to $\left(H_{1},(,)_{1}\right)$, such that

1) $D_{F} \subset H_{1}$ and $(F f, g)=(f, g)_{1}$ for $f \in D_{F}$ and $g \in H_{1}$

2) $\|f\|^{2} \leqq C(F f, f)$ for $f \in D_{F}$

3) $D_{F} \subset H_{1}$ is dense with respect to the norm \|\|$_{1}$ and $D_{F}=\left\{f \in H_{1} \mid \exists \tilde{f} \in H\right.$ s.t. $(f, g)_{1}=(\tilde{f}, g)$ for all $\left.g \in H_{1}\right\}, F f=\tilde{f}$.

We apply this theorem to the pair $\left\{L^{p, q}\left(X, E^{\otimes m}\right),(,)_{m}\right\}$ and $\left\{W^{p, q}\left(X, E^{\otimes m}\right)\right.$, $\left.Q_{m}(),\right\}$. Let $F_{m}$ be the Friedrichs operator associated to $\left\{W^{p, q}\left(X, E^{\otimes m}\right), Q_{m}(),\right\}$. The relation between the operators $\bar{\partial}, \bar{\partial}_{m}^{*}$ and $F_{m}$ on Hilbert space and the original ones $\bar{\partial}, \vartheta_{m}$ and $\square_{m}=\bar{\partial} \vartheta_{m}+\vartheta_{m} \bar{\partial}$ is as follows.

Proposition 2.3. 1) $C^{p, q}\left(\bar{X}, E^{\otimes m}\right)$ is dense in $D_{\overline{\bar{p}}}^{\underline{p}, q}$ with respect to the norm $\left(\|\|_{m}^{2}+\|\vec{\partial}\|_{m}^{2}\right)^{1 / 2}$.

2) For $1 \leqq s \leqq \infty, \quad B_{s}^{p, q}\left(\bar{X}, E^{\otimes m}\right)=C_{s}^{p, q}\left(\bar{X}, E^{\otimes m}\right) \cap D_{\bar{\partial}, q, q}^{p, q} \quad$ and $\quad \bar{\partial}_{m}^{*}=\vartheta_{m} \quad$ on $B_{s}^{p, q}\left(\bar{X}, E^{\otimes m}\right)$.

3) $B^{p, q}\left(\bar{X}, E^{\otimes m}\right)$ is dense in $D_{\bar{\partial}}^{p, q} \cap D_{\bar{\partial}}^{p, q}$ with respect to the norm $Q_{m}(,)^{1 / 2}$. In particular, $W^{p, q}\left(X, E^{\otimes m}\right)$ coincides with $D_{\bar{\partial}}^{p, q} \cap D_{\bar{\partial} *}^{p, q}$ in $L^{p, q}\left(X, E^{\otimes m}\right)$.

4) For $s \geqq 2, \quad D_{F m}^{p, q} \cap B_{s}^{p, q}\left(\bar{X}, E^{\otimes m}\right)=\left\{\varphi \in B_{s}^{p, q}\left(\bar{X}, E^{\otimes m}\right) \mid \bar{\partial} \varphi \in B_{s-1}^{p, q+1}\left(\bar{X}, E^{\otimes m}\right)\right\}$ and $F_{m}=\square_{m}+I$ on $D_{F m}^{p, q} \cap B_{s}^{p, q}\left(\bar{X}, E^{\otimes m}\right)$, where $I$ is the identity operator on $L^{p, q}\left(X, E^{\otimes m}\right)$.

Proof. 1) and 3) are due to Hörmander [4] Propositions 1.2.3 and 1.2.4. Combining 1) with the formula (2.9), we obtain 2). By Theorem 2.2, $F_{m}$ satisfies the equation $Q_{m}(\varphi, \phi)=\left(F_{m} \varphi, \phi\right)_{m}$ for $\varphi \in D_{F_{m}}^{p, q}$ and $\phi \in W^{p, q}\left(X, E^{\otimes m}\right)$. Hence using the formula (2.9), we obtain 4) (for a detail, see [2] (1.3.5) Proposition). 
In the end of this section, we give a sufficient condition for the solvability of the $L^{2} \bar{\partial}$-Neumann problem (for a detail, see [2] (3.1.14) Theorem and [7] p. 203-p. 213).

Theorem 2.4. In the short complex of Hilbert spaces

$$
L^{p, q-1}\left(X, E^{\otimes m}\right) \underset{\bar{\partial}_{m}^{*}}{\stackrel{\bar{\partial}}{\rightleftarrows}} L^{p, q}\left(X, E^{\otimes m}\right) \underset{\bar{\partial}_{m}^{*}}{\stackrel{\bar{\partial}}{\rightleftarrows}} L^{p, q+1}\left(X, E^{\otimes m}\right)
$$

if there exists a positive constant $C$, which may depend on $m$, such that

$$
\|\varphi\|_{m}^{2} \leqq C\left\{\|\bar{\partial} \varphi\|_{m}^{2}+\left\|\bar{\partial}_{m}^{*} \varphi\right\|_{m}^{2}\right\}
$$

if $\varphi \in D_{\bar{\partial}}^{p}, q \cap D_{\frac{p}{\partial}, q}^{p, q}$ and $\varphi \perp N_{\bar{\partial}}^{p}, q \cap N_{\bar{\partial}}^{p, q}$, , then it holds that

1) the operator $L_{m}=\bar{\partial} \bar{\partial}_{m}^{*}+\bar{\partial}_{m}^{*} \bar{\partial}: L^{p, q}\left(X, E^{\otimes m}\right) \rightarrow L^{p, q}\left(X, E^{\otimes m}\right)$ whose domain is

$$
D_{L_{m}}^{p, q}=\left\{\varphi \in D_{\bar{\partial}}^{p, q} \cap D_{\frac{p}{\bar{\partial}_{m}^{*}}, q}^{q} \mid \bar{\partial} \varphi \in D \frac{p, q+1}{\bar{\partial}_{m}^{*}} \text { and } \quad \bar{\partial}_{m}^{*} \varphi \in D_{\bar{\partial}}^{p, q-1}\right\}
$$

is self-adjoint, has a closed range and coincides with $F_{m}-I$ i.e. $D_{F m}^{p, q}=D_{L_{m}}^{p, q}$ and $L_{m}=F_{m}-I$.

2) there exists a unique bounded self-adjoint operator $N_{m}: L^{p, q}\left(X, E^{\otimes m}\right) \rightarrow$ $L^{p, q}\left(X, E^{\otimes m}\right)$, which is called the Neumann operator, such that

a) $D_{N_{m}}^{p, q}=L^{p, q}\left(X, E^{\otimes m}\right), \quad R_{N_{m}}^{p, q} \hookrightarrow D_{F_{m}}^{p, q}, \quad R_{N_{m}}^{p, q} \perp N_{L_{m}}^{p, q}$ and the nullity of $N_{m}$ coincides with $N_{L_{m}}^{p, q}$

b) for any $\alpha \in L^{p, q}\left(X, E^{\otimes m}\right)$

$$
\alpha=\bar{\partial} \bar{\partial}_{m}^{*} N_{m} \alpha+\bar{\partial}_{m}^{*} \bar{\partial} N_{m} \alpha+H_{m} \alpha
$$

where $H_{m}$ is the orthogonal projection onto $N_{L_{m}}^{p, q}$

c) $N_{m} L_{m}=L_{m} N_{m}=I-H_{m}$ on $D_{F m}^{p, q}$, and if $N_{m}$ is also defined on $L^{p, q+1}\left(X, E^{\otimes m}\right)$ (resp. $L^{p, q-1}\left(X, E^{\otimes m}\right)$ ), then $N_{m} \bar{\partial}=\bar{\partial} N_{m}$ on $D_{\bar{\partial}}^{p, q}$ (resp. $N_{m} \bar{\partial}_{m}^{*}=\bar{\partial}_{m}^{*} N_{m}$ on $\left.D \bar{\partial}_{m}^{p, q}\right)$

d) a necessary and sufficient condition for the existence of a solution $u$ satisfying $\bar{\partial} u=\alpha$ is that $\bar{\partial} \alpha=0$ and $\alpha \perp N_{L_{m}}^{p, q}$, then $u=\bar{\partial}_{m}^{*} N_{m} \alpha$

e) if $P: L^{p, q-1}\left(X, E^{\otimes m}\right) \rightarrow N_{\bar{\partial}}^{p, q-1}$ is the orthogonal projection onto $N_{\frac{p}{\partial}}, q-1$, then $P=I-\bar{\partial}_{m}^{*} N_{m} \bar{\partial}$ on $D \frac{p}{\bar{\partial}}, q-1$.

Remark 2.12. The nullity $N_{L}^{p, q}$ of $L_{m}$ always coincides with the space $N \frac{p}{\partial}, q \cap N \frac{p, q}{\partial}{ }_{m}^{*}$ without (2.11).

We say that the $L^{2} \bar{\partial}$-Neumann problem for $E^{\otimes m}$-valued forms of type $(p, q)$ on $X$ is solvable if we can prove the existence of the operator $N_{m}$ satisfying the conditions of Theorem 2.4, 2). Later we shall solve the $L^{2} \bar{\partial}$-Neumann problem on pseudoconvex domains by means of establishing the estimate (2.11).

\section{$\S 3$. Statement of Main Results}

Before mentioning our main results, we must prepare two definitions. Let $X$ be a relatively compact domain on an $n$-dimensional complex manifold $M$. 
Definition 3.1. $X$ is said to be a pseudoconvex domain with smooth boundary $\partial X$ if there exist a neighborhood $\Omega$ of $X$ and a real valued $C^{\infty}$-function $h$ on $\Omega$ such that 1) $\Omega \cap X=\{x \in \Omega\{h(x)<0\}$ and the gradient of $h$ nowhere vanishes on $\partial X 2)$ the complex Hessian of $h$ is positive semi-definite when restricted to the complex tangent space of $\partial X$.

Definition 3.2. A holomorphic line bundle $E \stackrel{\pi}{\rightarrow} M$ is said to be positive on a subset $Y$ of $M$ if there exist a coordinate cover $\left\{U_{i}\right\}_{i \in I}$ of $M$ such that $\pi^{-1}\left(U_{i}\right)$ are trivial and a hermitian metric $\boldsymbol{a}=\left\{a_{i}\right\}$ along the fibres of $E$ such that $-\log a_{i}$ is strictly plurisubharmonic on $U_{i} \cap Y$ for any $i \in I$.

Our main results are stated as follows.

Main results. Let $X$ be a pseudoconvex domain with smooth boundary $\partial X$ on an n-dimensional complex manifold $M$ and let $E$ be a holomorphic line bundle on $M$ which is positive on a neighborhood of $\partial X$. Then the following theorems $\mathrm{N}, \mathrm{R}_{s, \mu}$ and $\mathrm{N}_{s}$ hold for any non-negative integer $s$ and non-negative real number $\mu$.

Theorem $\mathbf{N}$. There exists a positive integer $m_{*}$ such that the $L^{2} \bar{\partial}$-Neumann problem for $E^{\otimes m}$-valued forms of type $(p, q)$ on $X$ is solvable in the sense of Theorem $2.4,2)$ for any $m \geqq m_{*}, p \geqq 0$ and $q \geqq 1$.

Theorem $\mathbf{R}_{s, \mu}$. There exists a positive integer $m(s, \mu) \geqq m_{*}$ depending on $s$ and $\mu$ such that the following statements $\mathrm{I}_{m, \lambda}^{s}, \mathrm{II}_{m, \lambda}^{s}$ and $\mathrm{III}_{m, \mu}$ hold for any $n \geqq$ $m(s, \mu)$ and $0 \leqq \lambda \leqq \mu$.

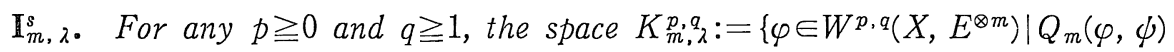
$-\lambda(\varphi, \phi)_{m}=0$ for any $\left.\phi \in W^{p, q}\left(X, E^{\otimes m}\right)\right\}$ is a finite dimensional subspace of $\mathcal{C}_{s}^{p, q}\left(\bar{X}, E^{\otimes m}\right)$ and $H_{m, \lambda}\left(\mathcal{C}_{s}^{p, q}\left(\bar{X}, E^{\otimes m}\right)\right) C_{\rightarrow} \mathcal{C}_{s}^{p, q}\left(\bar{X}, E^{\otimes m}\right)$ where $H_{m, \lambda}$ is the orthogonal projection onto the spaces $K_{m, \lambda}^{\cdot, \lambda}$.

$\mathrm{II}_{m, \lambda}^{s}$. For any $p \geqq 0$ and $q \geqq 1$, if $\alpha$ is an element of $\mathcal{C}_{s}^{p, q}\left(\bar{X}, E^{\otimes m}\right)$ such that $\alpha \perp K_{m, \lambda}^{p, q}$, then there exists a unique element $\varphi$ of $\mathcal{C}_{s}^{p, q}\left(\bar{X}, E^{\otimes m}\right) \cap K_{m, \lambda}^{p, q}$ such that

$$
Q_{m}(\varphi, \psi)-\lambda(\varphi, \psi)_{m}=(\alpha, \psi)_{m} \quad \text { for any } \phi \in W^{p, q}\left(X, E^{\otimes m}\right)
$$

and

$$
\|\bar{\partial} \varphi\|_{s}^{2}+\left\|\bar{\partial}_{m}^{*} \varphi\right\|_{s}^{2}+\|\varphi\|_{s}^{2} \leqq C_{m, s}(1+\lambda)^{s+1}\left\{\|\alpha\|_{s}^{2}+\|\varphi\|^{2}\right\}
$$

where $C_{m, s}$ is a positive constant depending on $m$ and $s$.

$\mathbf{I I I}_{m, \mu}$. The spectrum of the self-adjoint operator $L_{m}=\bar{\partial} \bar{\partial}_{m}^{*}+\bar{\partial}_{m}^{*} \bar{\partial}$ in the interval $[0, \mu]$ consists of finitely many eigenvalues.

In particular, we can solve the $\bar{\partial}$-Neumann problem satisfying the required global boundary regularity as follows.

Theorem $\mathbb{N}_{s}$. There exists a positive integer $m(s) \geqq m_{*}$ depending on $s$ such that for any $m \geqq m(s), p \geqq 0$ and $q \geqq 1$, it holds that

i) the space of harmonic forms $N_{L m}^{p, q}$ is a finite dimensional subspace of 
$\mathcal{C}_{s}^{p, q}\left(\bar{X}, E^{\otimes m}\right)$ and $H_{m}\left(\mathcal{C}_{s}^{p, q}\left(\bar{X}, E^{\otimes m}\right)\right) \subset_{\rightarrow} \mathcal{C}_{s}^{p, q}\left(\bar{X}, E^{\otimes m}\right)$ where $H_{m}$ is the orthogonal projection onto the spaces $N_{\ddot{L}_{m}}$

ii) the Neumann operator $N_{m}$ defined in Theorem $\mathrm{N}$ satisfies that $N_{m}, \bar{\partial} N_{m}$ and $\bar{\partial}_{m}^{*} N_{m} \operatorname{map} \mathcal{C}_{s}^{p, q}\left(\bar{X}, E^{\otimes m}\right)$ into $\mathcal{C}_{s}^{p, q}\left(\bar{X}, E^{\otimes m}\right), \mathcal{C}_{s}^{p, q+1}\left(\bar{X}, E^{\otimes m}\right)$ and $\mathcal{C}_{s}^{p, q-1}\left(\bar{X}, E^{\otimes m}\right)$ respectively and $\left\|N_{m} \alpha\right\|_{s}+\left\|\bar{\partial} N_{m} \alpha\right\|_{s}+\left\|\bar{\partial}_{m}^{*} N_{m} \alpha\right\|_{s} \leqq C_{m, s}^{\prime}\|\alpha\|_{s}$ for any $\alpha \in \mathcal{C}_{s}^{p, q}\left(\bar{X}, E^{\otimes m}\right)$, where $C_{m, s}^{\prime}$ is a positive constant depending on $m$ and $s$.

iii) $\mathcal{C}_{s}^{p, q-1}\left(\bar{X}, E^{\otimes m}\right) \cap N_{\bar{\partial}}^{p, q-1}$ is dense in $N_{\bar{\partial}}^{p, q-1}$.

Remark 3.3. Let $F$ be a line bundle on $M$. Then replacing $E^{\otimes m}$ by $E^{\otimes m} \otimes F$, we can prove Theorems $\mathrm{N}, \mathrm{R}_{s, \mu}$ and $\mathrm{N}_{s}$ for the line bundles $E^{\otimes m} \otimes F$. Since the proof of that case is quite parallel to the case $F$ is trivial, in this paper, we give only the proof of the case $F$ is trivial.

Remark 3.4. If there exists a strongly plurisubharmonic function $\Phi$ on a neighborhood $\Omega$ of $\partial X$, then any line bundle $E$ is positive on a relatively compact neighborhood of $\partial X$. In fact let $\boldsymbol{a}$ be a metric of $E$ on $M$ and extend $\Phi$ to a $C^{\infty}$-function $\Psi$ on $M$ without changing the original near $\partial X$ in a suitable manner. Then there exists a positive integer $m^{*}$ such that $\boldsymbol{a}_{m}=\boldsymbol{a} \exp (-m \Psi)$ gives the positivity of $E$ on a relatively compact neighborhood $\Omega^{\prime}(\Subset \Omega)$ of $\partial X$ for every $m \geqq m^{*}$. In this case, by changing the fibre metrics $\boldsymbol{a}_{m}$ of $E$ instead of taking the tensor product of $E$, we can set up the same problems for $E$ and can prove Theorems $\mathrm{N}, \mathrm{R}_{s, \mu}$ and $\mathrm{N}_{s}$ (see [6]). On the other hand, there are pseudoconvex domains with smooth boundary $\partial X$ not possessing such a strongly plurisubharmonic function on any neighborhood of $\partial X$ but possessing a line bundle which is positive on a neighborhood of $\partial X$ (see [3] [14]).

The practical merit of the regularization theorems $\mathrm{R}_{m, \mu}$ and $\mathrm{N}_{s}$ can be obtained by combining these theorems with Sobolev lemma (Lemma 2.1, 2)). Here we give only the detailed description of Theorem $\mathrm{N}_{s}$.

Corollary. We set ourselves in the situation of Theorem $\mathrm{N}_{s}$. If $s \geqq n+1$ and $m \geqq m(s)$, then we have the followings:

i) The operator $H_{m}$ maps $C^{p, q}\left(\bar{X}, E^{\otimes m}\right)$ into $C_{s-n-1}^{p, q}\left(\bar{X}, E^{\otimes m}\right)$. The operators $N_{m}, \bar{\partial} N_{m}$ and $\bar{\partial}_{m}^{*} N_{m}$ map $C^{p, q}\left(\bar{X}, E^{\otimes m}\right)$ into $C_{s-\frac{p}{n-1}}^{p,}\left(\bar{X}, E^{\otimes m}\right), C_{s-n-1}^{p, q+1}\left(\bar{X}, E^{\otimes m}\right)$ and

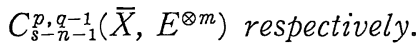

ii) For any element $\alpha$ of $C^{p, q}\left(\bar{X}, E^{\otimes m}\right)$ such that $\bar{\partial} \alpha=0$ and $\alpha \perp N_{L m}^{p, q}$, there exists an element $u$ of $C_{s-n}^{p, q-1}\left(\bar{X}, E^{\otimes m}\right)$ such that $u=\bar{\partial}_{m}^{*} N_{m} \alpha, \quad \bar{\partial} u=\alpha$ and $\|u\|_{s} \leqq$ $C_{m, s}^{\prime}\|\alpha\|_{s}$.

iii) $C_{s-n-1}^{p, q-1}\left(\bar{X}, E^{\otimes m}\right) \cap N_{\bar{\partial}}^{p, q-1}$ is dense in $N_{\bar{\partial}}^{p, q-1}$.

\section{§4. A Priori Estimates for Smooth Forms}

Let $X$ be a pseudoconvex domain with smooth boundary $\partial X$ on an $n$-dimensional complex manifold $M$. Let $E \stackrel{\pi}{\rightarrow} M$ be a holomorphic line bundle which is 
positive on a neighborhood $\Omega$ of $\partial X$. Let $\boldsymbol{a}=\left\{a_{\imath}\right\}$ be the metric of $E$ on $M$ which gives the positivity of $E$ on $\Omega$ with respect to a suitable covering $\left\{U_{\imath}\right\}_{i \in I}$ of $M$. Then the curvature form $\sum_{a, \beta=1}^{n}-\frac{\partial^{2} \log a_{i}}{\partial z_{\imath}^{\alpha} \partial z_{\imath}^{\bar{\beta}}} d z_{\imath}^{\alpha} \wedge d z_{\imath}^{\bar{\beta}}$ of $a$ provides a Kähler metric $d \sigma^{2}=\sum_{\alpha, \beta=1}^{n}-\frac{\partial^{2} \log a_{i}}{\partial z_{\imath}^{\alpha} \partial z_{\imath}^{\bar{\beta}}} d z_{\imath}^{\alpha} d z_{\imath}^{\bar{\beta}}$ on $\Omega$. We may assume that the defining function $h$ of $\partial X$ is constructed from the geodesic distance with respect to the metric $d \sigma^{2}$. Since using the function $h$, we can take a smooth product neighborhood of $\partial X$, we obtain the following lemma.

Lemma 4.1. Let $X$ and $E$ be as above. Then there exist neighborhoods $\Omega$ and $\Omega^{\prime}$ of $\partial X$, a coordinate covering $\left\{U_{i}\right\}_{i \in I}$ of $M$, a fibre metric $\boldsymbol{a}=\left\{a_{\imath}\right\}$ of $E$ on $M$ and a hermitian metric $d s^{2}=\sum_{\alpha, \beta=1}^{n} g_{i, \alpha \bar{\beta}} d z_{\imath}^{a} d z_{\imath}^{\bar{\beta}}$ on $M$ such that

1) $\Omega \Subset \Omega^{\prime}$ and $\bar{\Omega}^{\prime}$ is contained in a smooth product neighborhood of $\partial X$

2) $\pi^{-1}\left(\bar{U}_{i}\right)$ is trivial for any $i \in I$ and $U_{i} \Subset \Omega$ if $U_{i} \cap \partial X \neq \varnothing$

3) $E$ is positive on $\Omega^{\prime}$ with respect to $\boldsymbol{a}$

4) the restriction of $d s^{2}$ onto $\Omega^{\prime}$ coincides with the Kähler metric $d \sigma^{2}$.

From now on, we fix the above situation. With respect to the above metrics, we define the notations as in Section two. Let $U=\left\{U_{\imath}\right\}_{1 \leqq i \leqq N}$ be a finite covering of $\bar{X}$ in $\left\{U_{i}\right\}_{i \in I}$. We set $U_{1}=\left\{U_{i} \in \mathcal{U} \mid U_{i} \cap \partial X=\varnothing\right\}$ and $\mho_{2}=\left\{U_{i} \in \mathcal{U} \mid U_{i} \cap \partial X\right.$ $\neq \varnothing\}$. If $U_{\imath} \in U_{1}$, then we take a system of real coordinates $\left(x_{\imath}^{1}, \cdots, x_{\imath}^{2 n}\right)$ on $U_{i}$ such as taken in Section two and if $U_{i} \in \mathcal{U}_{2}$, then from Lemma 4.1,1) and 2), we can take a system of real coordinates $\left(t_{\imath}^{1}, \cdots, t_{\imath}^{2 n-1}, h\right)$ on $U_{i}$. With respect to these coordinates, $D_{2}^{\sigma}$ is defined for any multi-index $\sigma=\left(\sigma_{1}, \cdots, \sigma_{2 n}\right)$ as in section two. Let $\rho$ be a real-valued $C^{\infty}$-function on $M$ such that supp $\rho \Subset U_{i}$ for some $i \in\{1, \cdots, N\}$ and let $\left\{e_{i j}\right\}$ be the system of transition functions of $E$ with respect to $\left\{U_{i}\right\}_{i \in I}$. For a multi-index $\sigma=\left(\sigma_{1}, \cdots, \sigma_{2 n}\right)$, we define linear operators $\Delta_{l, \rho}^{\sigma}$ and $\Delta_{r, \rho}^{\sigma}: C^{p, q}\left(\bar{X}, E^{\otimes m}\right) \rightarrow C^{p, q}\left(\bar{X}, E^{\otimes m}\right)$ as follows : for $\varphi=\left\{\varphi_{i}\right\} \in C^{p, q}\left(\bar{X}, E^{\otimes m}\right)$ $\Delta_{l, \rho}^{\sigma}(\varphi)=\left\{\Delta_{l, \rho}^{\sigma}(\varphi)_{j}\right\}_{j \in I}$ (resp. $\left.\Delta_{r, \rho}^{\sigma}(\varphi)=\left\{\Delta_{r, \rho}^{\sigma}(\varphi)_{j}\right\}_{j \in I}\right)$ : if $j=i$, then $\Delta_{l, \rho}^{\sigma}(\varphi)_{i}=\rho D_{i}^{\sigma} \varphi_{i}$ (resp. $\left.\Delta_{r, \rho}^{\sigma}(\varphi)_{i}=D_{i}^{\sigma}\left(\rho \varphi_{i}\right)\right)$ on $U_{i}$, if $j \neq i$, then $\Delta_{l, \rho}^{\sigma}(\varphi)_{j}=e_{\imath j}^{-1} \cdot \Delta_{l, \rho}^{\sigma}(\varphi)_{i}$ on $U_{i} \cap U_{j}$ and 0 on $U_{\jmath} \backslash \operatorname{supp} \rho\left(\operatorname{resp} . \Delta_{r, \rho}^{\sigma}(\varphi)_{\jmath}=e_{\imath \jmath}^{-1} \cdot \Delta_{r, \rho}^{\sigma}(\varphi)_{i}\right.$ on $U_{i} \cap U_{j}$ and 0 on $U_{\jmath} \backslash \operatorname{supp} \rho$ ).

Here $D_{2}^{\sigma}$ acts on forms componentwise as in (2.5). In particular, if $\sigma=$ $(0, \cdots, 0)$, then we set $\Delta_{l, \rho}^{\sigma}=\Delta_{r, \rho}^{\sigma}=\Delta_{\rho}$. We define the formal adjoint $\Delta_{l, \rho, m}^{\sigma *}$ (resp. $\Delta_{r, \rho, m}^{\sigma *}$ ) of $\Delta_{l, \rho}^{\sigma}$ (resp. $\Delta_{r, \rho}^{\sigma}$ ) with respect to the inner product $(,)_{m}$ by the equation $\left(\Delta_{l, \rho}^{\sigma}(\varphi), \phi\right)_{m}=\left(\varphi, \Delta_{l, \rho, m}^{\sigma *}(\psi)\right)_{m} \quad$ (resp. $\left.\quad\left(\Delta_{r, \rho}^{\sigma}(\varphi), \psi\right)_{m}=\left(\varphi, \Delta_{r, \rho}^{\sigma *}(\psi)\right)_{m}\right)$ if $\varphi \in$ $C_{c}^{p, q}\left(X, E^{\otimes m}\right)$ or $\phi \in C_{c}^{p, q}\left(X, E^{\otimes m}\right)$. Especially, if $U_{i} \in \mathcal{U}_{2}$ i. e. $U_{i} \cap \partial X \neq \varnothing$, then for any multi-index $\sigma=\left(\sigma_{1}, \cdots, \sigma_{2 n}\right)$ so that $\sigma_{2 n}=0$, we can define the formal adjoint $\Delta_{l, \rho, m}^{\sigma *}$ (resp. $\Delta_{r, \rho, m}^{\sigma *}$ ) of $\Delta_{l, \rho}^{\sigma}$ (resp. $\left.\Delta_{r, \rho}^{\sigma}\right)$ on the spaces $C^{\cdot, \cdot}\left(\bar{X}, E^{\otimes m}\right)$ since $\Delta_{l, \rho}^{\sigma}$ (resp. $\Delta_{r, \rho}^{\sigma}$ ) does not contain the derivation with respect to $h$ and so, in view of Fubini's theorem, the boundary integral dose not appear by integration by parts. 
Lemma 4.2. If $U_{i} \in \bigcup_{2}$, then for any real-valued $C^{\infty}$-function $\rho$ on $M$ such that supp $\rho \Subset U_{i}$ and multi-index $\sigma=\left(\sigma_{1}, \cdots, \sigma_{2 n}\right)$ such that $\sigma_{2 n}=0$, it holds that $\Delta_{l, \rho}^{\sigma}\left(B^{p, q}\left(\bar{X}, E^{\otimes m}\right)\right) \hookrightarrow B^{p, q}\left(\bar{X}, E^{\otimes m}\right)$ and $\Delta_{r, \rho}^{\sigma}\left(B^{p, q}\left(\bar{X}, E^{\otimes m}\right)\right) \hookrightarrow B^{p, q}\left(\bar{X}, E^{\otimes m}\right) \quad$ (resp. $\Delta_{l, \rho, m}^{\sigma *}\left(B^{p, q}\left(\bar{X}, E^{\otimes m}\right)\right) \hookrightarrow B^{p, q}\left(\bar{X}, E^{\otimes m}\right)$ and $\left.\Delta_{\gamma, \rho}^{\sigma *}\left(B^{p, q}\left(\bar{X}, E^{\otimes m}\right)\right) \hookrightarrow B^{p, q}\left(\bar{X}, E^{\otimes m}\right)\right)(p \geqq 0$ and $q \geqq 1)$.

Proof. If necessary, shrinking $U_{i}$ arbitrarily, we can take a orthonormal basis $\left\{\omega_{1}, \cdots, \omega_{n}\right\}$ of $(1,0)$ forms on $U_{j}$ such that $\omega_{n}=\partial h$. We represent $\varphi \in$ $C^{p, q}\left(\bar{X}, E^{\otimes m}\right)$ with respect to this basis. Then $\varphi$ belongs to $B^{p, q}\left(\bar{X}, E^{\otimes m}\right)$ if and only if $\varphi_{j, J_{p}, K_{q}}=0$ on $U_{j} \cap \partial X$ for any $U_{j} \in \mathcal{U}_{2}$ whenever $n \in K_{q}=\left(k_{1}, \cdots, k_{q}\right)$ (see (2.10)). For any multi-index $K_{q}$ containing $n$, this implies that the defining function $h$ divides $\varphi_{i, J_{p}, \bar{K}_{q}}$ on $U_{i}$. For a multi-index $\sigma=\left(\sigma_{1}, \cdots, \sigma_{2 n}\right)$ such that $\sigma_{2 n}=0, D_{\imath}^{\sigma}$ does not contain the derivation with respect to $h$. Hence $h$ divides $\rho\left(D_{i} \varphi_{i, J_{p}, \bar{K}_{q}}\right)$ and $D_{i}\left(\rho \varphi_{i, J_{p}, \bar{K}_{q}}\right)$ on $U_{i}$. This means that $\Delta_{l, \rho}^{\sigma}(\varphi)$ and $\Delta_{r, \rho}^{\sigma}(\varphi)$ belong to $B^{p, q}\left(\bar{X}, E^{\otimes m}\right)$. Since $\Delta_{l, \rho, m}^{\sigma *}$ and $\Delta_{r, \rho, m}^{\sigma *}$ do not contain the derivation with respect to $h$, by the same way it is easily verified that $\Delta_{l, \rho, m}^{\sigma *}(\varphi)$ and $\Delta_{r, \rho, m}^{\sigma *}(\varphi)$ belong to $B^{p, q}\left(\bar{X}, E^{\otimes m}\right)$.

q. e. d.

From now on, we fix two families of real-valued $C^{\infty}$-functions $\left\{\rho_{i}\right\}_{1 \leqq i \leqq N}$ and $\left\{\zeta_{i}\right\}_{1 \leqq i \leqq N}$ such that $\rho_{i}, \zeta_{i} \in C_{c}^{0,0}\left(U_{i}\right), \zeta_{i} \equiv 1$ on $\operatorname{supp} \rho_{i}$ and $\sum_{i=1}^{N} \rho_{i} \equiv 1$ on $\bar{X}$. Using $\left\{\rho_{i}\right\}_{1 \leqq i \leqq N}$, we define the norms \|\|$_{s}$ as in Section 2. For real number $0<\delta \leqq 1$, we define the modified hermitian form $Q_{m, \delta}: B^{p, q}\left(\bar{X}, E^{\otimes m}\right) \times B^{p, q}\left(\bar{X}, E^{\otimes m}\right) \rightarrow C$ by $Q_{m, \delta}(\varphi, \psi)=Q_{m}(\varphi, \phi)+\delta(\varphi, \phi)_{m, 1} \quad$ where $\quad(\varphi, \phi)_{m, 1}=\sum_{i=1}^{N} \sum_{|\sigma| \leqq 1}\left(\Delta_{r, \zeta_{i}}^{\sigma}(\varphi), \Delta_{r, \zeta_{i}}^{\sigma}(\phi)\right)_{m}$. For any real number $\lambda$, we set

$$
Q_{m, \delta, \lambda}(\varphi, \psi)=Q_{m, \delta}(\varphi, \psi)-\lambda(\varphi, \psi)_{m} .
$$

Let $W_{\delta}^{p, q}\left(X, E^{\otimes m}\right)$ be the completion of $B^{p, q}\left(\bar{X}, E^{\otimes m}\right)$ under the norm $Q_{m, \delta}(,)^{1 / 2}$. Then it is clear that $W_{\delta}^{p, q}\left(X, E^{\otimes m}\right)$ is independent of $\delta$ and contained in $W^{p, q}\left(X, E^{\otimes m}\right) \cap \mathcal{C}_{1}^{p, q}\left(\bar{X}, E^{\otimes m}\right)$. Hence we set $W_{1}^{p, q}\left(X, E^{\otimes m}\right)=W_{\delta}^{p, q}\left(X, E^{\otimes m}\right)$ for any $0<\delta \leqq 1$. Then we can apply Theorem 2.2 to the Hilbert spaces $\left\{L^{p, q}\left(X, E^{\otimes m}\right)\right.$, $\left.(,)_{m}\right\}$ and $\left\{W_{1}^{p, q}\left(X, E^{\otimes m}\right), Q_{m, \delta}(),\right\}$. We denote by $F_{m, \delta}$ the Friedrichs operator associated to $\left\{W_{1}^{p, q}\left(X, E^{\otimes m}\right), Q_{m, \delta}(),\right\}$.

Let $\left\{\chi_{i}\right\}_{1 \leqq i \leqq N}$ and $\left\{\eta_{i}\right\}_{1 \leqq i \leq N}$ be real-valued $C^{\infty}$-functions on $M$ such that $\chi_{i}$, $\eta_{i} \in C_{c}^{0,0}\left(U_{i}\right), \chi_{i} \equiv 1$ on $\operatorname{supp} \rho_{i}$ and $\eta_{i} \equiv 1$ on supp $\chi_{i}$ for any $i$. The following lemma is essentially due to Kohn and Nirenberg (see Appendix I, Lemma A.1.1).

Lemma 4.3. For any $i \in\{1, \cdots, N\}, m \geqq 1$ and $s \geqq 0$, there exist positive constants $C_{s}$ and $C_{m, s}^{(k)}(k=1,2)$ such that

1) for any multi-index $\sigma=\left(\sigma_{1}, \cdots, \sigma_{2 n}\right)$ so that $|\sigma|=s$ if $U_{i} \in \mathcal{U}_{1},|\sigma|=s$ and $\sigma_{2 n}=0$ if $U_{i} \in \mathcal{U}_{2}$ and $\varphi \in B^{p, q}\left(\bar{X}, E^{\otimes m}\right)(p \geqq 0$ and $q \geqq 1)$, 


$$
\begin{aligned}
& Q_{m, \delta}\left(\Delta_{l, \rho_{i}}^{\sigma}(\varphi), \Delta_{l, \rho_{i}}^{\sigma}(\varphi)\right) \\
& \leqq \\
& \quad C_{s} \sum_{|\theta|=s}\left\|\Delta_{l, \chi_{i}}^{\theta}(\varphi)\right\|_{m}^{2}+2 \operatorname{Re} Q_{m, \delta}\left(\varphi, \Delta_{l, \rho_{i}, m}^{\sigma *} \Delta_{l, \rho_{i}}^{\sigma}(\varphi)\right) \\
& \quad+C_{m, s}^{(1)} \sum_{: \theta_{1} \leqq s-1}\left\|\Delta_{l, \eta_{i}}^{\theta}(\varphi)\right\|_{m}^{2} \\
& \quad+C_{m, s}^{(2)} \sum_{|\theta| \leq s-1} \operatorname{Re} Q_{m, \delta}\left(\varphi, \Delta_{l, x_{i}, m}^{\theta *} \Delta_{l, \chi_{i}}^{\theta}(\varphi)\right)
\end{aligned}
$$

2) $C_{s}$ (resp. $\left.C_{m, s}^{(k)}\right)$ depends on $s$ (resp. $m$ and $s$ ) and $C_{m, 0}^{(k)}=0$.

Under the situation of Lemma 4.1, we obtain the following estimate which is the consequence of a complex tensor calculus for Kähler manifolds with boundary (see Appendix II).

Proposition 4.4. There exist a positive constant $C$ not depending on $m$ and a positive integer $m_{0}$ such that for any $m \geqq m_{0}, p \geqq 0$ and $q \geqq 1$, if $\varphi \in B^{p, q}\left(\bar{X}, E^{\otimes m}\right)$, then

$$
\|\bar{\nabla} \varphi\|_{m, X \backslash K}^{2}+\left(m-m_{0}\right)\|\varphi\|_{m, X \backslash K}^{2} \leqq C\left\{\|\bar{\partial} \varphi\|_{m, X}^{2}+\left\|\bar{\partial}_{m}^{*} \varphi\right\|_{m, X}^{2}+\|\varphi\|_{m, K}^{2}\right\}
$$

where $K$ is the compact subset of $X$ defined by $K=X \backslash(X \cap \Omega)$ and $\bar{\nabla}$ is the covariant differentiation of type $(0,1)$ associated to the metric $d s^{2}$.

For $0 \leqq \delta \leqq 1, \lambda \geqq 0$ and $m \geqq 1$, we consider $\varphi \in B^{p, q}\left(\bar{X}, E^{\otimes m}\right) \cap D_{F m}^{p, q}\left(F_{m, 0}=F_{m}\right)$ and $\alpha \in C^{p, q}\left(\bar{X}, E^{\otimes m}\right)$ such that

$$
Q_{m, \delta, \lambda}(\varphi, \psi)=(\alpha, \psi)_{m} \quad \text { for } \phi \in B^{p, q}\left(\bar{X}, E^{\otimes m}\right), p \geqq 0 \text { and } q \geqq 1 \text { 。 }
$$

Let $s$ and $\mu$ be a non-negative integer and a non-negative real number respectively. Then we shall prove the following proposition.

Proposition 4.5. There exists a positive integer $m(s, \mu)$ such that the follow ing assertion $\mathrm{IV}_{m, \mu}^{s}$ holds for every $m \geqq m(s, \mu)$ and $0 \leqq \lambda \leqq \mu$.

$\mathbf{I V}_{m, \lambda}^{s}$. There exists a positive constant $C_{m, s}$ depending on $m$ and $s$ such that for any $\varphi \in B^{p, q}\left(\bar{X}, E^{\otimes m}\right) \cap D_{F_{m}^{\prime}, \delta}^{p, q}$ and $\alpha \in C^{p, q}\left(\bar{X}, E^{\otimes m}\right)$ which satisfy the equation $(4.3)_{\delta, \lambda}(p \geqq 0, q \geqq 1$ and $0 \leqq \delta \leqq 1)$

$$
\|\varphi\|_{s}^{2} \leqq C_{m, s}(1+\lambda)^{s}\left\{\|\alpha\|_{s}^{2}+\|\varphi\|^{2}\right\} .
$$

For the proof, we need the following two lemmas.

Lemma 4.6. If $U_{i} \in \mathcal{U}_{1}$, then the following assertion $\mathrm{V}_{m, \lambda}^{s}$ holds for every $m \geqq 1, s \geqq 0$ and $0 \leqq \lambda \leqq \mu$.

$\mathbf{V}_{m, \lambda}^{s}$. There exists a positive constant $C_{m, s+2}$ depending on $m$ and $s$ such that for any $\varphi \in B^{p, q}\left(\bar{X}, E^{\otimes m}\right) \cap D_{F_{m}, \delta}^{p, q}$ and $\alpha \in C^{p, q}\left(\bar{X}, E^{\otimes m}\right)$ which satisfy the equation $(4.3)_{\delta, \lambda}(p \geqq 0, q \geqq 1$ and $0 \leqq \delta \leqq 1)$

$$
\sum_{|\theta| \leqq s+2}\left\|\Delta_{l, \rho_{i}}^{\theta}(\varphi)\right\|_{m}^{2} \leqq C_{m, s+2}(1+\lambda)^{s+3}\left\{\sum_{|\theta| \leqq s}\left\|\Delta_{l, \eta_{i}}^{\theta}(\alpha)\right\|_{m}^{2}+\left\|\Delta_{\eta_{i}}(\varphi)\right\|_{m}^{2}\right\} .
$$


Lemma 4.7. If $U_{i} \in \mathcal{U}_{2}$, then the following assertion $\mathrm{VI}_{m, \lambda}^{s}$ holds for every $m \geqq m_{0}, s \geqq 0$ and $0 \leqq \lambda \leqq \mu$, where $m_{0}$ is the integer determined in Proposition 4.4.

$\mathrm{VI}_{m, \lambda}^{s}$. There exist positive constants $C_{s}$ and $C_{m, s}^{(k)}(k=1,2)$ such that

1) for any $\varphi \in B^{p, q}\left(\bar{X}, E^{\otimes m}\right) \cap D_{F, \delta}^{p, q}$ and $\alpha \in C^{p, q}\left(\bar{X}, E^{\otimes m}\right)$ which satisfy the equation $(4.3)_{j, \lambda}(p \geqq 0, q \geqq 1$ and $0 \leqq \delta \leqq 1)$

$$
\begin{gathered}
\left(m-m_{0}\right) \sum_{|\sigma| \leqq s}\left\|\Delta_{l, \rho_{i}}^{\sigma}(\varphi)\right\|_{m}^{2} \leqq(1+\lambda)\left\{C_{s} \sum_{\mid \theta_{1}=s}\left\|\Delta_{l, \eta_{i}}^{\theta}(\varphi)\right\|_{m}^{2}+C_{m, s}^{(1)} \sum_{|\theta| \leq s-1}\left\|\Delta_{l, \eta_{i}}^{\theta}(\varphi)\right\|_{m}^{2}\right\} \\
+C_{m, s}^{(2)}\left\{\sum_{\mid \theta_{1} \leqq s}\left\|\Delta_{l, \eta_{i}}^{\theta}(\alpha)\right\|_{m}^{2}+\left\|\Delta_{\eta_{i}}(\varphi)\right\|_{m}^{2}\right\}
\end{gathered}
$$

2) $C_{s}$ (resp. $\left.C_{m, s}^{(k)}\right)$ depends on $s$ (resp. $m$ and $s$ ) and $C_{m, 0}^{(1)}=0$.

We first prove the proposition by induction on $s$ using these lemmas.

Proof of the proposition. For $s=0$, setting $m(0, \mu)=1$, the proposition holds. By induction, suppose the proposition true for $s-1$. Let $m(s-1, \mu)$ be the integer determined by inductive hypothesis. Then for any integer $m \geqq$ $\max \left\{m(s-1, \mu), m_{0}\right\}$, using the fact $\eta_{i} \leqq C \sum_{j=1}^{N} \rho_{j}$ on $\bar{X}$ for some positive constant $C$ and inductive hypothesis, from (2.6), $\mathrm{V}_{m, \lambda}^{s-2}$ and $\mathrm{VI}_{m, \lambda}^{s}, 1$ ), we obtain the following. If $U_{i} \in \mathcal{U}_{1}$, then

$$
\left(m-m_{0}\right) \sum_{|\theta| \leqq s}\left\|\Delta_{l, \rho_{i}}^{\theta}(\varphi)\right\|_{m}^{2} \leqq C_{m, s}^{\prime}(1+\lambda)^{s+1}\left\{\|\alpha\|_{s}^{2}+\|\varphi\|^{2}\right\} .
$$

If $U_{i} \in \mathcal{U}_{2}$, then

$$
\begin{aligned}
\left(m-m_{0}\right) \sum_{|\theta| \leqq s}\left\|\Delta_{l, \rho_{i}}^{\theta}(\varphi)\right\|_{m}^{2} \leqq & (1+\lambda) C_{s} \sum_{j=1}^{N} \sum_{|\theta| \leqq s}\left\|\Delta_{l, \rho j}^{\theta}(\varphi)\right\|_{m}^{2} \\
& +C_{m, s}^{\prime}(1+\lambda)^{s+1}\left\{\|\alpha\|_{s}^{2}+\|\varphi\|^{2}\right\}
\end{aligned}
$$

where $C_{s}$ (resp. $C_{m, s}^{\prime}$ ) is a positive constant depending on $s$ (resp. $m$ and $s$ ). From (4.4) and (4.5), we have

$$
\begin{aligned}
\left(m-m_{0}\right) \sum_{i=1}^{N} \sum_{\mid \theta_{\mid} \leqq s}\left\|\Delta_{l, \rho_{i}}^{\theta}(\varphi)\right\|_{m}^{2} \leqq & (1+\lambda) N C_{s} \sum_{i=1}^{N} \sum_{\mid \theta_{\mid} \leqq s}\left\|\Delta_{l, \rho_{i}}^{\theta}(\varphi)\right\|_{m}^{2} \\
& +N C_{m, s}^{\prime}(1+\lambda)^{s+1}\left\{\|\alpha\|_{s}^{2}+\|\varphi\|^{2}\right\} .
\end{aligned}
$$

We determine an integer $m(s, \mu)$ as follows :

$$
m(s, \mu)=\max \left\{m(s-1, \mu),\left[(1+\mu) N C_{s}+\mu\right]+m_{0}+3\right\} .
$$

From (4.6), for any $m \geqq m(s, \mu)$, we have

$$
\sum_{i=1}^{N} \sum_{|\theta| \leqq s}\left\|\Delta_{r, \rho_{i}}^{\theta}(\varphi)\right\|_{m}^{2} \leqq C_{m, s}^{\prime \prime}\left\{(1+\lambda)^{s}\left(\|\alpha\|_{s}^{2}+\|\varphi\|^{2}\right)+\|\varphi\|_{s-1}^{2}\right\}
$$

for some positive constant $C_{m, s}^{\prime \prime}$ depending on $m$ and $s$. By inductive hypothesis, we obtain 


$$
\|\varphi\|_{s}^{2} \leqq C_{m, s}(1+\lambda)^{s}\left\{\|\alpha\|_{s}^{2}+\|\varphi\|^{2}\right\}
$$

for every $m \geqq i n(s, \mu)$ and a positive constant $C_{m, s}$ depending on $m$ and $s$. q.e.d.

Proof of Lemma 4.6. For simplicity, we omit the index $i$. To prove $\mathrm{V}_{m, \lambda}^{s}$, we prepare the assertion $\mathrm{VII}_{m}^{s}$ as follows. We set $\alpha_{*}=\alpha+\lambda \varphi$. Then the equation $(4.3)_{\delta, \lambda}$ can be written

$$
Q_{m, \delta}(\varphi, \psi)=\left(\alpha_{*}, \psi\right)_{m} \quad \text { for any } \psi \in B^{p, q}\left(\bar{X}, E^{\otimes m}\right) 。
$$

For $m \geqq 1$ and $s \geqq 0$, the assertion $\mathrm{VII}_{m}^{s}$ is described as follows:

$\mathrm{VII}_{m}^{s}$. For real-valued $C^{\infty}$-functions $\rho$ and $\eta$ such that $\rho, \eta \in C_{c}^{0,0}(U)$ and $\eta \equiv 1$ on supp $\rho$, there exists a positive constant $C_{m, s+2}$ depending on $m$ and $s$ such that for any $\varphi \in B^{p, q}\left(\bar{X}, E^{\otimes m}\right) \cap D_{F_{m}, \delta}^{p, q}$ and $\alpha_{*} \in C^{p, q}\left(\bar{X}, E^{\otimes m}\right)$ which satisfy the equation $(4.7)_{\delta}(p \geqq 0, q \geqq 1$ and $0 \leqq \delta \leqq 1)$,

$$
\sum_{\mid \theta_{1} \leqq s+2}\left\|\Delta_{l, \rho}^{\theta}(\varphi)\right\|_{m}^{2} \leqq C_{m, s+2}\left\{\sum_{\mid \theta_{1} \leqq s}\left\|\Delta_{l, \eta}^{\theta}\left(\alpha_{*}\right)\right\|_{m}^{2}+\left\|\Delta_{\eta}(\varphi)\right\|_{m}^{2}\right\} .
$$

This assertion is an immediate consequence of the coerciveness of $Q_{m, \delta}(0 \leqq$ $\delta \leqq 1$ ) on the spaces $C_{c}^{\cdot \cdot} \cdot\left(X, E^{\otimes m}\right)$ (for a detail, see [2] (2.5.5) Theorem). We prove Lemma 4.6 using VII $I_{m}^{s}$. Let $\left\{\rho_{k}\right\}_{0 \leqq k \leq t}(t=[s / 2]$ if $s$ is even, $t=[s / 2]+1$ if $s$ is odd) be real-valued $C^{\infty}$-functions on $M$ such that $\rho_{k} \in C_{c}^{0,0}(U), \rho_{0}=\rho, \rho_{t}=\eta$ and $\rho_{k+1} \equiv 1$ on supp $\rho_{k}(0 \leqq k \leqq t-1)$. Then applying $\mathrm{VII}_{m}^{s}$ repeatedly, $\mathrm{V}_{m, \lambda}^{s}$ can be obtained.

$$
\text { q.e.d. }
$$

Proof of Lemma 4.7. We first estimate the tangential derivatives. From now on, we omit the index $i$ for simplicity. If necessary, retaking the function $\eta$, we take real-valued $C^{\infty}$-functions $\left\{\rho_{k}\right\}_{1 \leqq k \leqq 4}$ on $M$ such that $\rho_{k} \in C_{c}^{0,0}(U), \rho_{1}=\rho$, $\rho_{4}=\eta$ and $\rho_{k} \equiv 1$ on supp $\rho_{k-1}(2 \leqq k \leqq 4)$. Let $\sigma=\left(\sigma_{1}, \cdots, \sigma_{2 n}\right)$ be a multi-index such that $|\sigma|=s$ and $\sigma_{2 n}=0$. Let $m \geqq m_{0}$. Then by Lemma 4.2, we have $\Delta_{l, \rho_{k}}^{\sigma}(\varphi) \in B^{p, q}\left(\bar{X}, E^{\otimes m}\right)$. From (4.2), we have

$$
\left(m-m_{0}\right)\left\|\Delta_{l, \rho_{2}}^{\sigma}(\varphi)\right\|_{m}^{2} \leqq Q_{m, \delta}\left(\Delta_{l, \rho_{2}}^{\sigma}(\varphi), \Delta_{l, \rho_{2}}^{\sigma}(\varphi)\right) .
$$

Applying (4.1), we have

$$
\begin{aligned}
\left(m-m_{0}\right)\left\|\Delta_{l, \rho_{2}}^{\sigma}(\varphi)\right\|_{m}^{2} \leqq & C_{s}^{\prime} \sum_{\mid \theta_{1}=s}\left\|\Delta_{l, \rho_{3}}^{\theta}(\varphi)\right\|_{m}^{2}+C_{m, s}^{\prime} \sum_{\mid \theta_{1 \leq s-1}}\left\|\Delta_{l, \rho_{4}}^{\theta}(\varphi)\right\|_{m}^{2} \\
& +2 \operatorname{Re} Q_{m, \delta}\left(\varphi, \Delta_{l, \rho_{2}, m}^{\sigma *} \Delta_{l, \rho_{2}}^{\sigma}(\varphi)\right) \\
& +C_{m, s}^{\prime \prime} \sum_{\mid \theta_{1}, \leq s-1} \operatorname{Re} Q_{m, \delta}\left(\varphi, \Delta_{l, \rho_{3}, m}^{\theta^{\prime} *} \Delta_{l, \rho_{3}}^{\theta^{\prime},}(\varphi)\right)
\end{aligned}
$$

where $C_{s}^{\prime}, C_{m, s}^{\prime}$ and $C_{m, s}^{\prime \prime}$ are positive constants such as taken in Lemma 4.3.

Combining $(4.3)_{\delta, \lambda}$ with Lemma 4.2 , we have

$$
Q_{m, \delta, \lambda}\left(\varphi, \Delta_{l, \rho_{k}, m}^{\theta *} \Delta_{l, \rho_{k}}^{\theta}(\varphi)\right)=\left(\Delta_{l, \rho_{k}}^{\theta}(\alpha), \Delta_{l, \rho_{k}}^{\theta}(\varphi)\right)_{m}
$$

for any multi-index $\sigma=\left(\sigma_{1}, \cdots, \sigma_{2 n}\right)$ such that $\sigma_{2 n}=0$ and $1 \leqq k \leqq 4$. Since the $2 n$-th components of $\theta^{\prime}$ and $\sigma$ of (4.8) are zero, combining (4.8) with (4.9), we 
have

$$
\begin{aligned}
\left(m-m_{0}\right)\left\|\Delta_{l, \rho_{2}}^{\sigma}(\varphi)\right\|_{m}^{2} \leqq & (1+\lambda)\left\{C_{s}^{\prime} \sum_{\mid \theta_{\mid}=s}\left\|\Delta_{l, \rho_{3}}^{\theta}(\varphi)\right\|_{m}^{2}+C_{m, s}^{\prime \prime} \sum_{\mid \theta_{1} \leqq s-1}\left\|\Delta_{l, \rho_{4}}^{\theta}(\varphi)\right\|_{m}^{2}\right\} \\
& +C_{m, s}^{\prime \prime}\left\{\sum_{\mid \theta_{\mid} \leq s}\left\|\Delta_{l, \rho_{3}}^{\theta}(\alpha)\right\|_{m}^{2}+\left\|\Delta_{\rho_{4}}(\varphi)\right\|_{m}^{2}\right\}
\end{aligned}
$$

for any multi-index $\sigma=\left(\sigma_{1}, \cdots, \sigma_{2 n}\right)$ such that $|\sigma|=s$ and $\sigma_{2 n}=0$.

Next we estimate the normal derivatives. We set $\sigma_{2 n}(t)=(0, \cdots, 0, t)$ for any non-negative integer $t$. Then we have

$$
\left\|\Delta_{r, \rho_{k}}^{\sigma_{2 n(1)}}(\varphi)\right\|_{m}^{2} \leqq C\left\{Q_{m, \hat{o}}\left(\Delta_{\rho_{k}}(\varphi), \Delta_{\rho_{k}}(\varphi)\right)+\sum_{\substack{\sigma \mid \leq 1 \leq 1 \\ \sigma_{2 n}=0}}\left\|\Delta_{l, \rho_{k+1}}^{\sigma}(\varphi)\right\|_{m}^{2}\right\}
$$

where $C$ is a positive constant not depending on $m$ and $1 \leqq k \leqq 3$. This can be seen as follows. Let $\left(z^{1}, \cdots, z^{n}\right)$ (resp. $\left(t^{1}, \cdots, t^{2 n-1}, h\right)$ ) be a system of holomorphic local coordinates (resp. the system of real local coordinates) on $U$. We set $w^{\nu}=t^{2 \nu-1}+\sqrt{-1} t^{2 \nu}(1 \leqq \nu \leqq n-1), w^{n}=t^{2 n-1}+\sqrt{-1} h, \partial Z={ }^{t}\left(\partial / \partial z^{1}, \cdots, \partial / \partial z^{n}\right), \partial W$ ${ }^{t}\left(\partial / \partial w^{1}, \cdots, \partial / \partial w^{n}\right)$ and $G=\left(g_{\alpha \bar{\beta}}\right)$. Then we have $\partial Z=J_{1} \partial W+J_{2} \overline{\partial W}$, where $J_{1}$ $=\left(\partial w^{\gamma} / \partial z^{\tau}\right)$ and $J_{2}=\left(\partial \bar{w}^{r} / \partial z^{\tau}\right)$ respectively. When we consider the quadratic form ${ }^{t} \overline{\partial Z^{t}} G^{-1} \partial Z$ as a polynomial of the variable $(\partial / \partial h)$, this quadratic form can be written as follows:

$$
\begin{aligned}
{ }^{t} \overline{\partial Z}^{t} G^{-1} \partial Z= & \frac{1}{4} b_{n \bar{n}}\left(\frac{\partial}{\partial h}\right)^{2}+\sum_{\nu=1}^{2 n-1} u_{2 n, \nu}\left(\frac{\partial}{\partial h}\right)\left(\frac{\partial}{\partial t^{\nu}}\right) \\
& +\sum_{\gamma, \nu=1}^{2 n-1} u_{\gamma, \nu}\left(\frac{\partial}{\partial t^{\gamma}}\right)\left(\frac{\partial}{\partial t^{\nu}}\right)
\end{aligned}
$$

where ${ }^{t}\left(J_{1}+J_{2}\right) G^{-1}\left(\overline{J_{1}+J_{2}}\right)=\left(b_{\alpha \bar{\beta}}\right)_{1 \leqq \alpha, \beta \leqq n}, u_{2 n, \nu}$ and $u_{\gamma, \nu}$ are $C^{\infty}$-functions on $U$ not depending on $m$.

Since the hermitian matrix ${ }^{t}\left(J_{1}+J_{2}\right) G^{-1}\left(\overline{J_{1}+J_{2}}\right)$ is positive definite over real numbers at each point of $U, b_{n} \bar{n}$ is a positive $C^{\infty}$-function on $U$. Hence, from (4.12) and Appendix II (A.2.2), we obtain

$$
\begin{gathered}
\left\|\Delta_{r, \rho_{k}}^{\sigma_{2 n(1)}}(\varphi)\right\|_{m}^{2}-\varepsilon C_{1}\left\|\Delta_{r, \rho_{k}}^{\sigma_{2 n(1)}}(\varphi)\right\|_{m}^{2}-\varepsilon^{-1} C_{2} \sum_{\substack{|\sigma| \leq 1 \\
\sigma_{2 n}=0}}\left\|\Delta_{r, \rho_{k}}^{\sigma}(\varphi)\right\|_{m}^{2} \\
\leqq C_{3}\left\|\bar{\nabla} \Delta_{\rho_{k}}(\varphi)\right\|_{m}^{2}
\end{gathered}
$$

for any $\varepsilon>0$ and positive constants $C_{1}, C_{2}$ and $C_{3}$ not depending on $\mathrm{m}$.

Combining (4.13) with (4.2), we obtain $(4.11)_{k}$ if $\varepsilon$ is small enough.

In $(4.11)_{2}$, replacing $\varphi$ by $\Delta_{l, \rho_{1}}^{\sigma}(\varphi)$ such that $\varphi \in B^{p, q}\left(\bar{X}, E^{\otimes m}\right),|\sigma| \leqq s-1$ and $\sigma_{2 n}=0$, we obtain

$$
\left\|\Delta_{l, \rho_{1}}^{\sigma+\sigma_{2 n}(1)}(\varphi)\right\|_{m}^{2} \leqq C\left\{Q_{m, \delta}\left(\Delta_{l, \rho_{1}}^{\sigma}(\varphi), \Delta_{l, \rho_{1}}^{\sigma}(\varphi)\right)+\sum_{\substack{\theta \mid \leq s \\ \theta_{2 n}=0}}\left\|\Delta_{l, \rho_{2}}^{\theta}(\varphi)\right\|_{m}^{2}\right\} .
$$

Applying (4.1) to the first term of the right-hand side of (4.14), for any multiindex $\sigma=\left(\sigma_{1}, \cdots, \sigma_{2 n}\right)$ such that $|\sigma| \leqq s-1$ and $\sigma_{2 n}=0$, we obtain 


$$
\begin{aligned}
\left\|\Delta_{l, \rho_{1}}^{\sigma+\sigma_{2} n(1)}(\varphi)\right\|_{m}^{2} \leqq & C \sum_{\substack{|\theta|=s \\
\theta_{2}=0}}\left\|\Delta_{l, \rho_{2}}^{\theta}(\varphi)\right\|_{m}^{2}+(1+\lambda) C_{m, s}^{\prime} \sum_{\mid \theta_{\mid} \leq s-1}\left\|\Delta_{l, \rho_{3}}^{\theta}(\varphi)\right\|_{m}^{2} \\
& +C_{m, s}^{\prime \prime}\left\{\sum_{\mid \theta_{1} \leq s}\left\|\Delta_{l, \rho_{2}}^{\theta}(\alpha)\right\|_{m}^{2}+\left\|\Delta_{\rho_{2}}(\varphi)\right\|_{m}^{2}\right\}
\end{aligned}
$$

where $C_{m, s}^{\prime}$ and $C_{m, s}^{\prime \prime}$ depend on $m$ and $s$.

Since $\alpha=\left(F_{m, \delta}-\lambda\right) \varphi$, using (4.12), $\alpha$ can be written as follows :

$$
\begin{aligned}
\alpha & =\left(\square_{m}+(I-\lambda)+\delta \sum_{|\sigma| \leq 1} \Delta_{r, \zeta, m}^{\sigma *} \Delta_{r_{0} \zeta}^{\sigma}\right) \varphi \\
& =-\left(\frac{1}{4} b_{n}-\delta \zeta^{2}\right)\left(\frac{\partial}{\partial h}\right)^{2} \varphi+\cdots .
\end{aligned}
$$

Since $A=(1 / 4) b_{n} \bar{n}+\delta \zeta^{2}$ is invertible on $U$, we obtain

$$
\begin{aligned}
& \left(\frac{\partial}{\partial h}\right)^{2} \varphi_{C_{p}, \bar{D}_{q}}=A^{-1}\left\{\left(\sum_{\nu=1}^{2 n-1} u_{2 n, \nu}^{\prime}\left(\frac{\partial}{\partial h}\right)\left(\frac{\partial}{\partial t^{\nu}}\right)+\sum_{\gamma, \nu=1}^{2 n-1} u_{\gamma, \nu}^{\prime}\left(\frac{\partial}{\partial t^{\gamma}}\right)\left(\frac{\partial}{\partial t^{\nu}}\right)\right.\right. \\
& \left.\left.+v_{m, 2 n}\left(\frac{\partial}{\partial h}\right)+\sum_{\gamma=1}^{2 n-1} v_{m, \gamma}\left(\frac{\partial}{\partial t^{\gamma}}\right)+v_{m, 0}\right) \varphi_{C_{p}, \bar{D}_{q}}-\alpha_{C_{p}, \bar{D}_{q}}\right\}
\end{aligned}
$$

where $u_{\gamma, \nu}^{\prime}(1 \leqq \gamma \leqq 2 n, 1 \leqq \nu \leqq 2 n-1)$ and $v_{m, \gamma}(0 \leqq \gamma \leqq 2 n)$ are $C^{\infty}$-functions on $U$ and $v_{m, r}$ depend on $m$.

The coefficients of the right-hand side of (4.16) depend on $\delta$. But they and their derivatives of higher order are uniformly bounded on any compact subset of $U$ with respect to $\delta$. For a multi-index $\sigma=\left(\sigma_{1}, \cdots, \sigma_{2 n}\right)$ such that $|\sigma| \leqq s-2$ and $\sigma_{2 n}=0$, we operate $D^{\sigma}$ on (4.16). Then using (4.15), $\left\|\Delta_{l, \rho_{1}}^{\sigma+\sigma_{2 n}(2)}(\varphi)\right\|_{m}^{2}$ can be dominated by the right-hand side of (4.15) for suitable constants $C, C_{m, s}^{\prime}$ and $C_{m, s}^{\prime \prime}$. By successive differentiations of (4.16) and proceeding similarly, $\sum_{t=1}^{s} \sum_{\mid \sigma_{1}=s-t}\left\|\Delta_{l, \rho_{1}}^{\sigma+\sigma_{2 n}(t)}(\varphi)\right\|_{m}^{2}$ can be dominated by the right-hand side of (4.15). Hence, for any $m \geqq m_{0}$, we obtain

$$
\begin{aligned}
\left(m-m_{0}\right) \sum_{\mid \theta_{1} \leqq s}\left\|\Delta_{l, \rho_{1}}^{\theta}(\varphi)\right\|_{m}^{2} \leqq & C^{\prime}\left(m-m_{0}\right) \sum_{\substack{\mid \theta_{1}=s \\
\theta_{2 n}=0}}\left\|\Delta_{l, \rho_{1}}^{\theta}(\varphi)\right\|_{m}^{2}+(1+\lambda) C_{m, s}^{\prime \prime} \sum_{|\theta| \leqq s-1}\left\|\Delta_{l, \rho_{4}}^{\theta}(\varphi)\right\|_{m}^{2} \\
& +C_{m, s}\left\{\sum_{\mid \theta_{1} \leqq s}\left\|\Delta_{l, \rho_{4}}^{\theta}(\alpha)\right\|_{m}^{2}+\left\|\Delta_{\rho_{4}}(\varphi)\right\|_{m}^{2}\right\}
\end{aligned}
$$

by (4.10)

$$
\begin{gathered}
\leqq(1+\lambda)\left\{C_{s} \sum_{|\theta|=s}\left\|\Delta_{l, \rho_{4}}^{\theta}(\varphi)\right\|_{m}^{2}+C_{m, s}^{(1)} \sum_{\mid \theta_{1} \leq s-1}\left\|\Delta_{l, \rho_{4}}^{\theta}(\varphi)\right\|_{m}^{2}\right\} \\
+C_{m, s}^{(2)}\left\{\sum_{\mid \theta_{1} \leqq s}\left\|\Delta_{l, \rho_{4}}^{\theta}(\alpha)\right\|_{m}^{2}+\left\|\Delta_{\rho_{4}}(\varphi)\right\|_{m}^{2}\right\}
\end{gathered}
$$

for positive constants $C_{s}, C_{m, s}^{(1)}$ and $C_{m, s .}^{(2)}$ Hence $\mathrm{VI}_{m, \lambda}^{s}$ has been proved. q. e. d.

Next we shall prove the following theorem.

Theorem 4.8. There exists a positive integer $m(s, \mu)$ such that the following assertion $\mathrm{VIII}_{m, \lambda}^{s}$ holds for every $m \geqq m(s, \mu)$ and $0 \leqq \lambda \leqq \mu$.

VIII ${ }_{m, \lambda}^{s}$. There exists a positive constant $C_{m, s}$ depending on $m$ and $s$ such that for any $\varphi \in B^{p, q}\left(\bar{X}, E^{\otimes m}\right) \cap D_{F, q}^{p, q}$ and $\alpha \in C^{p, q}\left(\bar{X}, E^{\otimes m}\right)$ which satisfy the equation $(4.3)_{\delta, \lambda}(p \geqq 0, q \geqq 1$ and $0 \leqq \delta \leqq 1)$ 
$\|\bar{\partial} \varphi\|_{s}^{2}+\left\|\bar{\partial}_{m}^{*} \varphi\right\|_{s}^{2}+\|\varphi\|_{s}^{2}+\delta\|\varphi\|_{s+1}^{2} \leqq C_{m, s}(1+\lambda)^{s+1}\left\{\|\alpha\|_{s}^{2}+\|\varphi\|^{2}\right\}$.

Proof. We prove the theorem by induction on $s$. Let $\left\{\eta_{i}\right\}_{1 \leqq i \leqq N}$ be realvalued $C^{\infty}$-functions on $M$ such that $\eta_{i} \in C_{c}^{0,0}\left(U_{i}\right)$ and $\eta_{i} \equiv 1$ on $\operatorname{supp} \zeta_{i}$ for any $i$.

For $s=0$, setting $m(0, \mu)=1$, the theorem holds. By induction, suppose the theorem true for $s-1$ and let $m(s-1, \mu)$ be the integer determined by inductive hypothesis. Then we can find an integer $m(s, \mu) \geqq m(s-1, \mu)$ such that $\mathrm{IV}_{m, \lambda}^{s}$ holds for any $m \geqq m(s, \mu)$. We fix an integer $m \geqq m(s, \mu)$ and an index $i \in$ $\{1, \cdots, N\}$. Let $\sigma=\left(\sigma_{1}, \cdots, \sigma_{2 n}\right)$ be a multi-index such that $|\sigma| \leqq s$ if $U_{i} \in \mathcal{U}_{1}$, $|\sigma| \leqq s$ and $\sigma_{2 n}=0$ if $U_{i} \in \mathcal{U}_{2}$. Using Proposition 2.3,4) and Lemma 4.2, we have

We set

$$
\begin{aligned}
\left\|\Delta_{l, \rho_{i}}^{\sigma} \bar{\partial} \varphi\right\|_{m}^{2}= & \left(\Delta_{l, \rho_{i}}^{\sigma} \bar{\partial}_{m}^{*} \bar{\partial} \varphi, \Delta_{l, \rho_{i}}^{\sigma}(\varphi)\right)_{m}+\left(\left[\bar{\partial}_{m}^{*}, \Delta_{l, \rho_{i}}^{\sigma}\right] \bar{\partial} \varphi, \Delta_{l, \rho_{i}}^{\sigma}(\varphi)\right)_{m} \\
& +\left(\Delta_{l, \rho_{i}}^{\sigma} \bar{\partial} \varphi,\left[\Delta_{l, \rho_{i}}^{\sigma}, \bar{\partial}\right] \varphi\right)_{m} .
\end{aligned}
$$

$$
A\left(\bar{\partial}, \bar{\partial}_{m}^{*}, \varphi\right)=\left(\left[\bar{\partial}_{m}^{*}, \Delta_{l, \rho_{i}}^{\sigma}\right] \bar{\partial} \varphi, \Delta_{l, \rho_{i}}^{\sigma}(\varphi)\right)_{m}+\left(\Delta_{l, \rho_{i}}^{\sigma} \bar{\partial} \varphi,\left[\Delta_{l, \rho_{i}}^{\sigma}, \bar{\partial}\right] \varphi\right)_{m} .
$$

Applying the same calculation to $\left\|\Delta_{l, \rho_{i}}^{\sigma} \bar{\partial}_{m}^{*} \varphi\right\|_{m}^{2}$ and $\left\|\Delta_{l, \rho_{i}}^{\sigma} \Delta_{r, \zeta_{i}}^{\theta}(\varphi)\right\|_{m}^{2}$, we obtain

$$
\begin{aligned}
(*)_{\rho_{i}}^{\sigma}:= & \left\|\Delta_{l, \rho_{i}}^{\sigma} \bar{\partial} \varphi\right\|_{m}^{2}+\left\|\Delta_{l, \rho_{i}}^{\sigma} \bar{\partial}_{m}^{*} \varphi\right\|_{m}^{2}+\left\|\Delta_{l, \rho_{i}}^{\sigma}(\varphi)\right\|_{m}^{2}+\delta \sum_{\mid \theta_{1} \leqq 1}\left\|\Delta_{l, \rho_{i}}^{\sigma} \Delta_{r, \zeta_{i}}^{\theta}(\varphi)\right\|_{m}^{2} \\
= & \left(\Delta_{l, \rho_{i}}^{\sigma}(\alpha), \Delta_{l, \rho_{i}}^{\sigma}(\varphi)\right)_{m}+\lambda\left\|\Delta_{l, \rho_{i}}^{\sigma}(\varphi)\right\|_{m}^{2}+A\left(\bar{\partial}, \bar{\partial}_{m}^{*}, \varphi\right) \\
& +A\left(\bar{\partial}_{m}^{*}, \bar{\partial}, \varphi\right)+\delta \sum_{\mid \theta_{1} \leqq 1} A\left(\Delta_{r, \zeta_{i}}^{\theta}, \Delta_{r, \zeta_{i}, m}^{\theta *}, \varphi\right) .
\end{aligned}
$$

Using Appendix I, (A.1.5), we obtain

$$
(*)_{\rho_{i}}^{\sigma} \leqq\left\|\Delta_{l, \rho_{i}}^{\sigma}(\alpha)\right\|_{m}^{2}+\varepsilon^{-1} C_{m, s}^{(1)}(1+\lambda) \sum_{\left|\sigma^{\prime}\right| \leq s}\left\|\Delta_{l, \eta_{i}}^{\sigma^{\prime}}(\varphi)\right\|_{m}^{2}+\varepsilon C_{m, s}^{(2)} \sum_{\left|\sigma^{\prime}\right| \leqq s}(*)_{\eta_{i}}^{\sigma^{\prime}}
$$

for positive constants $C_{m, s}^{(1)}, C_{m, s}^{(2)}$ and any $\varepsilon>0$. Using $\mathrm{IV}_{m, \lambda}^{s}$, we obtain

$$
\begin{aligned}
(*)_{\rho_{i}}^{\sigma} \leqq & C_{m, s}^{(3)}\left\{\varepsilon^{-1}(1+\lambda)^{s+1}\left(\|\alpha\|_{s}^{2}+\|\varphi\|^{2}\right)\right. \\
& \left.+\varepsilon\left(\|\bar{\partial} \varphi\|_{s}^{2}+\left\|\bar{\partial}_{m}^{*} \varphi\right\|_{s}^{2}+\|\varphi\|_{s}^{2}+\delta\|\varphi\|_{s+1}^{2}\right)\right\} \cdots(* *)_{\varepsilon}
\end{aligned}
$$

for any $\varepsilon>0$ and a positive constant $C_{m, s}^{(3)}$.

Next we estimate the normal derivatives. Let $\sigma=\left(\sigma_{1}, \cdots, \sigma_{2 n}\right)$ be a multiindex such that $|\sigma|=s-1$ and $\sigma_{2 n}=0$. Using (4.16), we have

$$
\delta\left\|\Delta_{l, \rho_{i}}^{\sigma+\sigma_{2 n(1)}} \Delta_{r, \zeta_{i}}^{\sigma_{2 n}(1)}(\varphi)\right\|_{m}^{2} \leqq C_{m, s}^{(4)}\left\{\sum_{\substack{\left|\sigma^{\prime}\right| \leq s \\ \sigma_{2 n}=0}}\left\|\Delta_{l, \rho_{i}}^{\sigma^{\prime}}(\varphi)\right\|_{m}^{2}+\delta \sum_{\substack{|\theta| \leq 1 \\\left|\sigma^{\prime}\right| \leq s, \sigma_{2 n}^{\prime}=0}}\left\|\Delta_{l, \rho_{i}}^{\sigma^{\prime}} \Delta_{r, \zeta_{i}}^{\theta}(\varphi)\right\|_{m}^{2}\right\}
$$

$$
\leqq(* *)_{\varepsilon} .
$$

By successive differentiations of (4.16) and proceeding similarly, we obtain

$$
\delta \sum_{\substack{1 \sigma \mid=s-k \\ 0 \leqq k \leqq s}}\left\|\Delta_{l, \rho_{i}}^{\sigma+\sigma_{2 n}(k)} \Delta_{r, \zeta_{i}}^{\sigma_{2 n}(1)}(\varphi)\right\|_{m}^{2} \leqq(* *)_{\varepsilon} .
$$

We consider the operator $L: C^{p, q-1}\left(\bar{X}, E^{\otimes m}\right) \oplus C^{p, q+1}\left(\bar{X}, E^{\otimes m}\right) \rightarrow C^{p, q}\left(\bar{X}, E^{\otimes m}\right) \oplus$ $C^{p, q+2}\left(\bar{X}, E^{\otimes m}\right)$ defined by $L(\varphi, \psi)=\left(\bar{\partial} \varphi+\vartheta_{m} \psi, \bar{\partial} \psi\right)$. It is clear that $L$ is elliptic 
and of first order. Hence the normal derivatives $(\partial / \partial h)(\varphi, \psi)$ can be written as a linear combination of $L(\varphi, \psi)$ and the tangential derivatives of $(\varphi, \psi)$. Let $\sigma$ be a multi-index such that $|\sigma|=s-1$ and $\sigma_{2 n}=0$. Since $L\left(\bar{\partial}_{m}^{*} \varphi, \bar{\partial} \varphi\right)=(\alpha+(\lambda-1)$ $\left.-\delta \sum_{i=1}^{N} \sum_{\mid \theta_{1} \leqq 1} \Delta_{r, \zeta_{i}, m}^{\theta *} \Delta_{r, \zeta_{i}}^{\theta}(\varphi), 0\right)$, using (4.18) and $\operatorname{IV}_{m, \lambda}^{s}$, we obtain

$$
\left\|\Delta_{l, \rho_{i}}^{\sigma+\sigma_{2 n}(1)} \bar{\partial} \varphi\right\|_{m}^{2}+\left\|\Delta_{l, \rho_{i}}^{\sigma+\sigma_{2 n}(1)} \bar{\partial}_{m}^{*} \varphi\right\|_{m}^{2} \leqq(* *)_{\varepsilon} .
$$

By successive differentiations of $(\partial / \partial h)\left(\bar{\partial}_{m}^{*} \varphi, \bar{\partial} \varphi\right)$ and proceeding similarly, we obtain

$$
\sum_{\substack{\sigma \mid=s-k \\ 0 \leq k \leq s}}\left\|\Delta_{l, \rho_{i}}^{\sigma+\sigma_{2 n}(k)} \bar{\partial} \varphi\right\|_{m}^{2}+\left\|\Delta_{l, \rho_{i}}^{\sigma+\sigma_{2 n}(k)} \bar{\partial}_{m}^{*} \varphi\right\|_{m}^{2} \leqq(* *)_{s} .
$$

Hence, from (4.17), (4.18) and (4.19), $\sum_{i=1}^{N} \sum_{|\sigma| \leqq s}(*)_{\rho_{i}}^{\sigma}$ can be estimated by $(* *)_{\varepsilon}$. Hence by inductive hypothesis and $\mathrm{IV}_{m, \lambda}^{s}$, we have

$$
\begin{aligned}
& \|\bar{\partial} \varphi\|_{s}^{2}+\left\|\bar{\partial}_{m}^{*} \varphi\right\|_{s}^{2}+\|\varphi\|_{s}^{2}+\delta\|\varphi\|_{s+1}^{2} \\
& \quad \leqq C_{m, s}^{(5)}\left\{\varepsilon^{-1}(1+\lambda)^{s+1}\left(\|\alpha\|_{s}^{2}+\|\varphi\|^{2}\right)+\varepsilon\left(\|\bar{\partial} \varphi\|_{s}^{2}+\left\|\bar{\partial}_{m}^{*} \varphi\right\|_{s}^{2}+\|\varphi\|_{s}^{2}+\delta\|\varphi\|_{s+1}^{2}\right)\right\}
\end{aligned}
$$

for any $\varepsilon>0$.

Therefore we obtain $\mathrm{VIII}_{m, \lambda}^{s}$ if $\varepsilon$ is small enough.

Remark. In preparation of this paper, the author knew that such a priori estimate as the above type had been obtained by D. Catlin (see [1]).

\section{$\S 5$. Proof of Main Results}

Throughout this section, we set ourselves in the same situation as Section 4.

Proposition 5.1. i) There exists a positive integer $m_{*}$ such that for any $m \geqq m_{*}$

1) the space $N_{\frac{\partial}{\partial}}^{p, q} \cap N_{\frac{p}{\partial} * q}^{p}$, has finite dimension for $p \geqq 0$ and $q \geqq 1$

2) there exists a positive constant $C_{m}$ depending on $m$ such that

$$
\|\varphi\|_{m} \leqq C_{m}\left\{\|\bar{\partial} \varphi\|_{m}^{2}+\left\|\bar{\partial}_{m}^{*} \varphi\right\|_{m}^{2}\right\}
$$

if $\varphi \in D_{\bar{\partial}}^{p, q} \cap D_{\bar{\partial}}^{p, q}, \varphi \perp N_{\bar{\partial}}^{p}, q \cap N \frac{p, q}{\bar{\partial} *}, p \geqq 0$ and $q \geqq 1$.

ii) For any real number $\mu \geqq 0$, there exists a positive integer $m(\mu)$ depending on $\mu$ such that for any $m \geqq m(\mu)$ and $0 \leqq \lambda \leqq \mu$

1) the space $K_{m, \lambda}^{p, q_{2}}=\left\{\varphi \in W^{p, q}\left(X, E^{\otimes m}\right) \mid Q_{m, \lambda}(\varphi, \psi)=0\right.$ for any $\left.\psi \in W^{p, q}\left(X, E^{\otimes m}\right)\right\}$ has finite dimension for $p \geqq 0$ and $q \geqq 1$

2) there exists a positive constant $C_{m, \lambda}$ depending on $m$ and $\lambda$ such that

$$
\|\varphi\|_{m} \leqq C_{m, \lambda}\left\|\left(F_{m}-\lambda\right) \varphi\right\|_{m}
$$

if $\varphi \in D_{F}^{p, q}, \varphi \perp K_{m}^{p, q}, p \geqq 0$ and $q \geqq 1$. 
iii) For any $m \geqq 1,0<\delta \leqq 1$ and $\lambda \geqq 0$, it holds that

1) the space $K_{m, \delta, \lambda}^{p, q_{i}}=\left\{\varphi \in W_{1}^{p, q}\left(X, E^{\otimes m}\right) \mid Q_{m, \delta, \lambda}(\varphi, \phi)=0\right.$ for any $\phi \in$ $\left.W_{1}^{p, q}\left(X, E^{\otimes m}\right)\right\}$ has finite dimension for $p \geqq 0$ and $q \geqq 1$

2) there exists a positive constant $C_{m, \delta, \lambda}$ depending on $m, \delta$ and $\lambda$ such that

$$
\|\varphi\|_{m} \leqq C_{m, \delta, \lambda}\left\|\left(F_{m, \delta}-\lambda\right) \varphi\right\|_{m}
$$

if $\varphi \in D_{F}^{p, q}, \dot{\delta}, \varphi \perp K_{m, \delta, \lambda}^{p, q}, p \geqq 0$ and $q \geqq 1$.

Proof. We first prove the assertions i) and ii). Let $m_{0}$ and $C$ be the positive integer and the positive constant determined in Proposition 4.4 respectively. Then we determine two positive integers $m_{*}$ and $m(\mu)$ as follows

$$
m_{*}=m_{0}+1 \text { and } m(\mu)=[C \mu]+m_{*} .
$$

Let $\chi$ be a real-valued $C^{\infty}$-function on $M$ such that supp $\chi \subset X$ and $\chi \equiv 1$ on $K$, where $K$ is the compact subset of $X$ determined in Proposition 4.4. Then, from (4.2), we obtain the following two estimates:

$(5.4)$ i) If $m \geqq m_{*}$ and $\varphi \in B^{p, q}\left(\bar{X}, E^{\otimes m}\right)$, then

$$
\|\varphi\|_{m}^{2} \leqq C_{m}\left\{D_{m}(\varphi, \varphi)+\left\|\chi_{\varphi}\right\|_{m}^{2}\right\}
$$

where $C_{m}$ is a positive constant depending on $m$.

ii) If $m \geqq m(\mu), 0 \leqq \lambda \leqq \mu$ and $\varphi \in B^{p, q}\left(\bar{X}, E^{\otimes m}\right)$, then

$$
\|\varphi\|_{m}^{2} \leqq C_{m, \mu}\left\{Q_{m, \lambda}(\varphi, \varphi)+\|\chi \varphi\|_{m}^{2}\right\}
$$

where $C_{m, \mu}$ is a positive constant depending on $m$ and $\mu$. The assertions i) and ii) are derived from the above estimates i) and ii) respectively. Since the proof of i) is similar to [13] Proposition 1.11, we give only the proof of ii). We fix an integer $m \geqq m(\mu)$ and a real number $\lambda \in[0, \mu]$. To show 1$)$, we have only to prove that $K_{m, \lambda}^{p, q}$ is locally compact. In view of Proposition 2.3,3), let $\left\{\varphi_{\nu}\right\}_{\nu \geqq 1}$ be a sequence of $B^{p, q}\left(\bar{X}, E^{\otimes m}\right)$ such that $\left\|\varphi_{\nu}\right\|_{m} \leqq 1$ and $Q_{m, \lambda}\left(\varphi_{\nu}, \varphi_{\nu}\right) \rightarrow 0$ as $\nu \rightarrow \infty$. Then $\left(\square_{m}\left(\chi_{\varphi_{\nu}}\right), \chi_{\varphi_{\nu}}\right)_{m}+\left(\chi_{\varphi_{\nu}}, \chi_{\varphi_{\nu}}\right)_{m}=Q_{m}\left(\chi_{\varphi_{\nu}}, \chi_{\varphi_{\nu}}\right)$ is bounded since $Q_{m}\left(\varphi_{\nu}, \varphi_{\nu}\right)$ is bounded. Hence combining Gårding's inequality (see [2] (2.2.1) Theorem) with Lemma 2.1, 1), there exists a subsequence of $\left\{\chi_{\varphi_{\nu}}\right\}_{\nu \geqq 1}$ which is Cauchy in $L^{p, q}\left(X, E^{\otimes m}\right)$. On the other hand, the inequality of (5.4), ii) implies that if $\left\{\chi_{\varphi_{\nu}}\right\}_{\nu \geqq 1}$ is Cauchy in $L^{p, q}\left(X, E^{\otimes m}\right)$, then $\left\{\varphi_{\nu}\right\}_{\nu \geqq 1}$ is Cauchy in $L^{p, q}\left(X, E^{\otimes m}\right)$. Combining this fact with the above argument, we obtain that $K_{m, \lambda}^{p, q}$ is locally compact. To prove 2), we assume that the assertion were false. Then there would be a sequence $\left\{\varphi_{\nu}\right\}_{\nu \geqq 1}$ such that $\varphi_{\nu} \in D_{F_{m}}^{p, q},\left\|\varphi_{\nu}\right\|_{m}=1$ and $\left\|\left(F_{m}-\lambda\right) \varphi_{\nu}\right\|_{m} \rightarrow 0$ as $\nu \rightarrow \infty$. Combining Proposition 2.3,3) with the proof of 1), taking a subsequence, we may assume that $\left\{\varphi_{\nu}\right\}_{\nu \geqq 1}$ is Cauchy in $L^{p, q}\left(X, E^{\otimes m}\right)$. Hence $\left\{\varphi_{\nu}\right\}_{\nu \geqq 1}$ is Cauchy in $W^{p, q}\left(X, E^{\otimes m}\right)$. Let $\varphi=\lim _{\nu \rightarrow \infty} \varphi_{\nu}$ in $W^{p, q}\left(X, E^{\otimes m}\right)$. Then we have $\varphi \in K_{m, \lambda}^{p, q_{2}}$ and $\|\varphi\|_{m}=1$. On the other hand, we have $\varphi \perp K_{m, 2}^{p, q_{2}}$. This is a contradiction. The assertion iii) is caused by the coercive estimate $\|\varphi\|_{m, 1}^{2} \leqq$ 
$\delta^{-1} Q_{m, \delta}(\varphi, \varphi)$ on the spaces $B^{\cdot \cdot \cdot}\left(\bar{X}, E^{\otimes m}\right)$. By Lemma $\left.2.1,1\right)$, this estimate implies that any $Q_{m, \delta}($,$) -bounded sequence has a Cauchy subsequence in L^{p, q}\left(X, E^{\otimes m}\right)$. Hence the proof of iii) can be done similarly to ii).

q. e. d.

Proof of Theorems N ( $\left.=\mathrm{N}_{0}\right)$ and $\mathrm{R}_{0, \mu}$. First, combining Theorem 2.4 with Proposition 5.1, i), 2), we obtain Theorem N. Next, we fix a non-negative real number $\mu$ and prove Theorem $\mathrm{R}_{0, \mu}$. We set $m(0, \mu)=m(\mu)$, where $m(\mu)$ is the integer determined in Proposition 5.1, ii). For any $m \geqq m(0, \mu)$ and $0 \leqq \lambda \leqq \mu, I_{m, \lambda}^{0}$ follows from Proposition 5.1, ii), 1). $\mathrm{II}_{m, \lambda}^{0}$ is derived as follows. Let $\alpha$ be an element of $L^{p, q}\left(X, E^{\otimes m}\right)$ such that $\alpha \perp K_{m, \lambda}^{p, q}$. From (5.2), we have $\left|(\alpha, \phi)_{m}\right| \leqq$ $C_{m, \lambda}\|\alpha\|_{m}\left\|\left(F_{m}-\lambda\right) \psi\right\|_{m}$ for every $\phi \in D_{F m}^{p, q}$. This implies that there exists a unique element $\varphi$ of $L^{p, q}\left(X, E^{\otimes m}\right)$ such that $(\alpha, \psi)_{m}=\left(\varphi,\left(F_{m}-\lambda\right) \psi\right)_{m}$ for any $\phi \in D_{F_{m}^{\prime}}^{p, q}$ Hence we have $\varphi \in D_{F m}^{p, q} \cap K_{m, \lambda}^{p, q \perp}, \alpha=\left(F_{m}-\lambda\right) \varphi$ and $\|\varphi\|_{m} \leqq C_{m, \lambda}\|\alpha\|_{m}$. $\operatorname{III}_{m, \mu}$ is derived from a fundamental fact of spectral theory for operators on Hilbert spaces. Let $\lambda_{0}$ be an eigenvalue of $F_{m}$ in $[0, \mu]$ i. e. $\operatorname{dim}_{C} K_{m, 2}^{p, q}>0$. Combining Proposition 5.1, ii), 2) with Appendix III, Theorem A.3.2, we obtain that $\lambda_{0}$ is isolated. Since the spectrum of $F_{m}$ is closed in the real line, the eigenvalues of $F_{m}$ contained in $[0, \mu]$ consists of finitely many points. Combining Theorem 2.4 , 1) with Proposition 5.1, i), 2), we have $F_{m}=L_{m}+I$. Hence we obtain $\operatorname{III}_{m, \mu}$. q. e. d.

Proof of Theorem $\mathrm{R}_{s, \mu}(s \geqq 1$ and $\mu \geqq 0)$. For the proof, we need the following regularization theorem.

Theorem $\mathbf{R}_{\delta}(\mathbf{0}<\boldsymbol{\delta} \leqq \mathbf{1})$. For any $m \geqq 1, \lambda \geqq 0, p \geqq 0$ and $q \geqq 1$,

1) if $\left(F_{m, \delta}-\lambda\right) \theta=0$, then $\theta \in B^{p, q}\left(\bar{X}, E^{\otimes m}\right) \cap D_{F, \delta}^{p, q}$

2) if $\alpha$ is an element of $C^{p, q}\left(\bar{X}, E^{\otimes m}\right)$ such that $\alpha \perp K_{m, \delta, \lambda}^{p, q}$, then there exists a unique element $\varphi_{\delta}$ of $B^{p, q}\left(\bar{X}, E^{\otimes m}\right) \cap D_{F_{m}, \delta}^{p, q} \cap K_{m, \delta, \lambda}^{p, q}$ such that $Q_{m, \delta, \lambda}\left(\varphi_{\delta}, \psi\right)=$ $(\alpha, \psi)_{m}$ for any $\phi \in W_{1}^{p, q}\left(X, E^{\otimes m}\right)$.

This regularization theorem is derived from the coerciveness of the modified hermitian form $Q_{m, \delta}$ on the spaces $B^{\cdot \cdot \cdot(}\left(\bar{X}, E^{\otimes m}\right)$ (for a detail, see [2] p. 31-p. 35, 3. Elliptic regularization, p. 47 and p. 48 (3.1.1), (3.1.2) Propositions and (3.1.3) Corollary).

Given $s \geqq 1$ and $\mu \geqq 0$, we take the integer $m(s, \mu)$ determined in Theorem 4.8 and fix an integer $m \geqq m(s, \mu)$. Since we may assume $m(s, \mu) \geqq m(0, \mu), \operatorname{III}_{m, \mu}$ of Theorem $\mathrm{R}_{s, \mu}$ follows from $\mathrm{III}_{m, \mu}$ of Theorem $\mathrm{R}_{0, \mu}$. Let $\Sigma_{m, \mu}$ be the set of eigenvalues of $F_{m}$ in $[0, \mu]$. Then $\Sigma_{m, \mu}$ is a finite point set. We set $\Lambda_{m, \mu}=$ $[0, \mu] \backslash \Sigma_{m, \mu}$. The proof of the assertions $\mathrm{I}_{m, \lambda}^{s}$ and $\mathrm{II}_{m, \lambda}^{s}$ is separated into two cases.

The case $\lambda \in \Lambda_{m, \mu}$.

Since $K_{m, \lambda}^{p, q}=\{0\}, \mathrm{I}_{m, \lambda}^{s}$ is clear. To prove $\mathrm{II}_{m, \lambda}^{s}$, we first prove the following assertion : 
(5.5) There exists a positive constant $\delta_{0}$ such that $K_{m, \delta, \lambda}^{p, q}=\{0\}$ for every $p \geqq 0$, $q \geqq 1$ and $0 \leqq \delta \leqq \delta_{0}$.

If (5.5) were false, then there would be sequences $\left\{\delta_{\nu}\right\}_{\nu \geq 1}$ and $\left\{\theta_{\nu}\right\}_{\nu \geqq 1}$ such that $\delta_{\nu} \rightarrow 0$ as $\nu \rightarrow \infty, \quad \theta_{\nu} \in K_{m, \delta_{\nu}, \lambda}^{p, q}$ and $\left\|\theta_{\nu}\right\|_{m}=1$. Since $\theta_{\nu} \in B^{p, q}\left(\bar{X}, E^{\otimes m}\right) \cap D_{F_{m}, \delta_{\nu}}^{p, q}$ by Theorem $\mathrm{R}_{\delta_{\nu}}, 1$ ), from Theorem 4.8 , VIII ${ }_{m, \lambda}^{s}$, we obtain $\left\|\theta_{\nu}\right\|_{s}^{2} \leqq C_{m, s}(1+\lambda)^{s+1}\left\|\theta_{\nu}\right\|^{2}$ for any $\nu \geqq 1$. Since $s \geqq 1$, at least $\left\|\theta_{2}\right\|_{1}$ is bounded. From Lemma 2.1,1) and the equation $Q_{m, \delta_{\nu}, \lambda}\left(\theta_{\nu}, \theta_{\nu}\right)=0$, taking a subsequence, we can conclude that $\left\{\theta_{\nu}\right\}_{\nu \geqq 1}$ converges strongly to an element $\theta$ of $W^{p, q}\left(X, E^{\otimes m}\right)$ with respect to the norm $Q_{m}(,)^{1 / 2}$. Moreover we have $Q_{m, \lambda}(\theta, \phi)=0$ for any $\phi \in W^{p, q}\left(X, E^{\otimes m}\right)$ since $\left\|\theta_{\nu}\right\|_{m, 1}$ is bounded. Hence $\theta \in K_{m, \lambda}^{p, q}=\{0\}$. On the other hand, $\|\theta\|_{m}=1$. This is a contradiction. Hence (5.5) has been proved.

Next we prove the following assertion $\mathrm{II}_{m, \delta, \lambda}^{s}\left(0<\delta \leqq \delta_{0}\right.$ and $\left.m \geqq m(s, \mu)\right)$.

$\mathbf{I}_{m, \delta, \lambda}^{s}$. For any $p \geqq 0$ and $q \geqq 1$, if $\alpha$ is an element of $\mathcal{C}_{s}^{p, q}\left(\bar{X}, E^{\otimes m}\right)$, then there exists a unique element $\varphi_{\delta}$ of $\mathcal{C}_{\delta+1}^{p, q}\left(\bar{X}, E^{\otimes m}\right) \cap D_{F m, \delta}^{p, q}$ such that $Q_{m, \delta, \lambda}\left(\varphi_{\delta}, \phi\right)$ $=(\alpha, \phi)_{m}$ for any $\phi \in W_{1}^{p, q}\left(\bar{X}, E^{\otimes m}\right)$ and

$$
\left\|\bar{\partial} \varphi_{\delta}\right\|_{s}^{2}+\left\|\bar{\partial}_{m}^{*} \varphi_{\delta}\right\|_{s}^{2}+\left\|\varphi_{\delta}\right\|_{s}^{2} \leqq C_{m, s}(1+\lambda)^{s+1}\left\{\|\alpha\|_{s}^{2}+\left\|\varphi_{\delta}\right\|^{2}\right\}
$$

where $C_{m, s}$ is the positive constant determined in $\mathrm{VIIII}_{m, \lambda}^{s}$.

Proof of $\mathrm{II}_{m, \delta, \lambda}^{s}$. Using Proposition 5.5, iii) and (5.5), the existence and uniqueness of $\varphi_{o}$ in $D_{F_{m}, \delta}^{p, q}$ can be proved similarly to $\mathrm{II}_{m, \lambda}^{0}$ of Theorem $\mathrm{R}_{0, \lambda}$. On the other hand, there exists a sequence $\left\{\alpha_{\nu}\right\}_{\nu \geq 1}$ of $C^{p, q}\left(\bar{X}, E^{\otimes m}\right)$ such that $\left\|\alpha_{\nu}-\alpha\right\|_{s \rightarrow 0}$ as $\nu \rightarrow \infty$. From Theorem $\mathrm{R}_{\delta}$ and (5.5), we obtain that there exists a unique element $\varphi_{\delta, \nu}$ of $B^{p, q}\left(\bar{X}, E^{\otimes m}\right) \cap D_{F_{m, \delta}^{, q}}^{p, q}$ such that $Q_{m, \delta, \lambda}\left(\varphi_{\delta, \nu}, \psi\right)=\left(\alpha_{\nu}, \psi\right)_{m}$ for any $\phi \in W_{1}^{p, q}\left(X, E^{\otimes m}\right)$. We apply $\operatorname{VIII}_{m, \lambda}^{s}$ to $\varphi_{\delta, \nu}$ and $\varphi_{\delta, \nu_{1}}-\varphi_{\delta, \nu_{2}}$ respectively. Then we have

$$
\begin{aligned}
& \left\|\bar{\partial} \varphi_{\delta, \nu}\right\|_{s}^{2}+\left\|\bar{\partial}_{m}^{*} \varphi_{\delta, \nu}\right\|_{s}^{2}+\left\|\varphi_{\delta, \nu}\right\|_{s}^{2} \leqq C_{m, s}(1+\lambda)^{s+1}\left\{\|\alpha\|_{s}^{2}+\left\|\varphi_{\delta, \nu}\right\|^{2}\right\} \\
& \delta\left\|\varphi_{\delta, \nu_{1}}-\varphi_{\delta, \nu_{2}}\right\|_{s+1}^{2} \leqq C_{m, s}(1+\lambda)^{s+1}\left\{\left\|\alpha_{\nu_{1}}-\alpha_{\nu_{2}}\right\|_{s}^{2}+\left\|\varphi_{\delta, \nu_{1}}-\varphi_{\delta, \nu_{2}}\right\|^{2}\right\}
\end{aligned}
$$

Combining (5.3) with (5.7), we obtain that there exists an element $\varphi_{\delta}^{*}$ of $\mathcal{C}_{s+1}^{p, q}\left(\bar{X}, E^{\otimes m}\right)$ such that $\left\|\varphi_{\delta, \nu}-\varphi_{\delta}^{*}\right\|_{s+1} \rightarrow 0$ as $\nu \rightarrow \infty$. Since $s \geqq 1$, we have $Q_{m, \delta, \lambda}\left(\varphi_{\delta}^{*}, \psi\right)=(\alpha, \psi)_{m}$ for any $\psi \in W_{1}^{p, q}\left(X, E^{\otimes m}\right)$. By uniqueness, we have $\varphi_{\delta}^{*}=\varphi_{\delta}$ in $W_{1}^{p, q}\left(X, E^{\otimes m}\right)$ and so $\varphi_{\delta}^{*}=\varphi_{\delta}$ in $\mathcal{C}_{s+1}^{p, q}\left(\bar{X}, E^{\otimes m}\right)$. Finally, from (5.6), we obtain $\mathrm{II}_{m, \delta, \lambda}^{s}$.

q. e. d.

Proof of $\mathrm{II}_{m, \lambda}^{s}$. Let $\alpha$ be an element of $\mathcal{C}_{s}^{p, q}\left(\bar{X}, E^{\otimes m}\right)$ and let $\varphi$ be the solution of the equation $\left(F_{m}-\lambda\right) \varphi=\alpha$ taken in $\mathrm{II}_{m, \lambda}^{0}$ of Theorem $\mathrm{R}_{0, \mu}$. Let $\varphi_{\delta}$ be the solution of the equation $\left(F_{m, \delta}-\lambda\right) \varphi_{\delta}=\alpha$ taken in $\mathrm{II}_{m, \delta, \lambda}^{s}\left(0<\delta \leqq \delta_{0}\right)$. Then we assert the following:

$$
\left\{\left\|\varphi_{\delta}\right\|_{m}\right\}_{0<\delta \leq \delta_{0}} \text { is bounded. }
$$

If (5.8) were false, then there would be a sequence $\left\{\delta_{\nu}\right\}_{\nu \geqq 1}$ such that $\delta_{\nu} \rightarrow 0$ and 
$\left\|\varphi_{\delta_{\nu}}\right\|_{m} \rightarrow \infty$ as $\nu \rightarrow \infty$. Setting $\xi_{\nu}=\varphi_{\delta_{\nu}} /\left\|\varphi_{\delta_{\nu}}\right\|_{m}$, from $I_{m, \delta, \lambda}^{s}$, it follows that $\left\|\xi_{\nu}\right\|_{s}$ is bounded. Hence, from Lemma $2.1,1)$ and the equation $Q_{m, \delta_{\nu}, \lambda}\left(\xi_{\nu}, \xi_{\nu}\right)=$ $\left(\alpha, \xi_{\nu}\right) /\left\|\varphi_{\delta_{\nu}}\right\|_{m}$, taking a subsequence, we can conclude that $\left\{\xi_{\nu}\right\}_{\nu \geqq 1}$ converges strongly to an element $\xi$ of $W^{p, q}\left(X, E^{\otimes m}\right)$ with respect to the norm $Q_{m}(,)^{1 / 2}$. Moreover we have $Q_{m, \lambda}(\xi, \phi)=0$ for any $\phi \in W^{p, q}\left(X, E^{\otimes m}\right)$ since $Q_{m, \delta_{\nu}, \lambda}\left(\xi_{\nu}, \eta\right)=$ $(\alpha, \eta)_{m} /\left\|\varphi_{\delta_{\nu}}\right\|_{m}$ for any $\eta \in B^{p, q}\left(\bar{X}, E^{\otimes m}\right)$. Hence $\xi \in K_{m, \lambda}^{p, q}=\{0\}$. On the other hand, $\|\xi\|_{m}=1$. This is a contradiction. Hence (5.8) has been proved.

Let $\left\{\delta_{\nu}\right\}_{\nu \geq 1}$ be a sequence such that $0<\delta_{\nu} \leqq \delta_{0}$ and $\delta_{\nu} \rightarrow 0$ as $\nu \rightarrow \infty$. Then combining $\mathrm{II}_{m, \delta_{\nu}, \lambda}^{s}$ with (5.8), we obtain that $\left\|\bar{\partial} \varphi_{\delta_{\nu}}\right\|_{s},\left\|\bar{\partial}_{m}^{*} \varphi_{\delta_{\nu}}\right\|_{s}$ and $\left\|\varphi_{\delta_{\nu}}\right\|_{s}$ are bounded. From Lemma 2.1,1) and the equation $Q_{m, \delta, \lambda}\left(\varphi_{\delta}, \varphi_{\delta}\right)=\left(\alpha, \varphi_{\delta}\right)_{m}$, taking a subsequence, we obtain that there exists an element $\varphi_{*}$ of $\mathcal{C}_{s}^{p, q}\left(\bar{X}, E^{\otimes m}\right) \cap$ $W^{p, q}\left(X, E^{\otimes m}\right)$ such that $\left\{\varphi_{\delta_{\nu}}\right\}_{\nu \geq 1}$ converges strongly to $\varphi_{*}$ with respect to the norm $Q_{m}(,)^{1 / 2}$ and converges weakly to $\varphi_{*}$ in $\mathcal{C}_{s}^{p, q}\left(\bar{X}, E^{\otimes m}\right)$. Hence we have $Q_{m, \lambda}\left(\varphi_{*}, \psi\right)=(\alpha, \psi)_{m}$ for any $\phi \in W^{p, q}\left(X, E^{\otimes m}\right)$. By uniqueness, we have $\varphi_{*}=\varphi$ in $W^{p, q}\left(X, E^{\otimes m}\right)$ and so they coincide with in $\mathcal{C}_{s}^{p, q}\left(\bar{X}, E^{\otimes m}\right)$. Since we may assume that $\left\{\varphi_{\delta_{\nu}}\right\},\left\{\bar{\partial} \varphi_{\delta_{\nu}}\right\}$ and $\left\{\bar{\partial}_{m}^{*} \varphi_{\delta_{\nu}}\right\}$ converge weakly to $\varphi, \bar{\partial} \varphi$ and $\bar{\partial}_{m}^{*} \varphi$ in $\mathcal{C}_{\dot{s}}^{*} \cdot\left(\bar{X}, E^{\otimes m}\right)$ respectively, taking a subsequence of $\left\{\varphi_{\delta_{\nu}}\right\}$, we can conclude that the arithmetic means of them converge strongly to $\varphi, \bar{\partial} \varphi$ and $\bar{\partial}_{m}^{*} \varphi$ in $\mathcal{C}_{\dot{s}^{\prime}} \cdot\left(\bar{X}, E^{\otimes m}\right)$ respectively. Hence from $\mathrm{II}_{m, \delta_{\nu}, \lambda}^{s}$, we obtain the desired inequality of $\mathrm{II}_{m, \lambda}^{s}$. Hence in the case $\lambda \in \Lambda_{m, \mu}, \mathrm{I}_{m, \lambda}^{s}$ and $\mathrm{II}_{m, \lambda}^{s}$ have been proved completely.

The case $\lambda \in \Sigma_{m, \mu}$.

First we prove $\mathrm{I}_{m, \lambda}^{s}$. From Proposition 5.1, ii), 1 ), $K_{m, \lambda}^{p, q}$ is a finite dimensional subspace of $L^{p, q}\left(X, E^{\otimes m}\right)$. To show $K_{m, \lambda}^{p, q_{\lambda}} \subseteq \mathcal{C}_{s}^{p, q}\left(\bar{X}, E^{\otimes m}\right)$, we proceed by induction. Let $k$ be an integer such that $0 \leqq k<\operatorname{dim}_{C} K_{m, \lambda}^{p, q}$ and $\theta_{1}, \cdots, \theta_{k}$ are $k$ linearly independent vectors in $K_{m}^{p, q} \cap \mathcal{C}_{s}^{p, q}\left(\bar{X}, E^{\otimes m}\right)$. We will construct another vector $\theta$ in $K_{m, 2}^{p, q} \cap \mathcal{C}_{s}^{p, q}\left(\bar{X}, E^{\otimes m}\right)$ such that $\|\theta\|_{m}=1$ and $\left(\theta, \theta_{j}\right)_{m}=01 \leqq j \leqq k$. (If $k=0$, we simply construct a non-zero vector $\theta$ in $K_{m, 2}^{p, q} \cap \mathcal{C}_{s}^{p, q}\left(\bar{X}, E^{\otimes m}\right)$.) As the dimension of $K_{m, \lambda}^{p, q_{2}}$ is finite, this will show $K_{m, \lambda}^{p, q} \mathcal{C}_{>} \mathcal{C}_{s}^{p, q}\left(\bar{X}, E^{\otimes m}\right)$. We can suppose without restriction that $\theta_{1}, \cdots, \theta_{k}$ are orthonormal. Let $\omega$ be an element of $K_{m, \lambda}^{p, q}$ such that $\theta_{1}, \cdots, \theta_{k}$ and $\omega$ are still orthonormal. Then since $C^{p, q}\left(\bar{X}, E^{\otimes m}\right)$ is dense in $L^{p, q}\left(X, E^{\otimes m}\right)$, there exists an element $\alpha$ of $\mathcal{C}_{s}^{p, q}\left(\bar{X}, E^{\otimes m}\right)$ such that $\left(\alpha, \theta_{j}\right)_{m}=0$ for $1 \leqq j \leqq k$ and $(\alpha, \omega)_{m} \neq 0$. Next we take a sequence $\left\{\lambda_{\nu}\right\}_{\nu \geq 1}$ of $\Lambda_{m, \mu}$ such that $\lambda_{\nu} \uparrow \lambda$ as $\nu \rightarrow \infty$. From $\mathrm{II}_{m, \lambda^{\prime}}^{s}$ for the case $\lambda^{\prime} \in \Lambda_{m, \mu}$, there exists a sequence $\left\{\varphi_{\nu}\right\}_{\nu \geqq 1}$ of $\mathcal{C}_{s}^{p, q}\left(\bar{X}, E^{\otimes m}\right) \cap D_{F m}^{p, q}$ such that for any $\nu \geqq 1$,

$$
\begin{gathered}
Q_{m, \lambda_{\nu}}\left(\varphi_{\nu}, \psi\right)=(\alpha, \psi)_{m} \quad \text { for } \phi \in W^{p, q}\left(X, E^{\otimes m}\right) \\
\left\|\bar{\partial} \varphi_{\nu}\right\|_{s}^{2}+\left\|\bar{\partial}_{m}^{*} \varphi_{\nu}\right\|_{s}^{2}+\left\|\varphi_{\nu}\right\|_{s}^{2} \leqq C_{m, s}(1+\lambda)^{s+1}\left\{\|\alpha\|_{s}^{2}+\left\|\varphi_{\nu}\right\|^{2}\right\} .
\end{gathered}
$$

Then we assert the following:

$$
\left\{\left\|\varphi_{\nu}\right\|_{m}\right\}_{\nu \geqq 1} \text { is unbounded. }
$$

If it were bounded, then from (5.10), $\left\|\varphi_{\nu}\right\|_{s}$ is bounded. Then we can construct 
an element $\varphi$ of $W^{p, q}\left(X, E^{\otimes m}\right)$ such that $Q_{m, \lambda}(\varphi, \psi)=(\alpha, \psi)_{m}$ for any $\phi \in$ $W^{p, q}\left(X, E^{\otimes m}\right)$ (see the proof of $\mathrm{II}_{m, \lambda}^{s}$ for the case $\lambda \in \Lambda_{m, \mu}$ ). In particular, replacing $\phi$ by $\omega$, we have $(\alpha, \omega)_{m}=0$ since $\omega \in K_{m}^{p, q}$. This contradicts to the choice of $\alpha$. Hence (5.11) has been proved. If necessary, taking a subsequence, we may assume that $\left\|\varphi_{\nu}\right\|_{m} \rightarrow \infty$ as $\nu \rightarrow \infty$. Setting $\beta_{\nu}=\varphi_{\nu} /\left\|\varphi_{\nu}\right\|_{m}$, from (5.10), it follows that $\left\|\beta_{\nu}\right\|_{s}$ is bounded. Then we can construct an element $\theta$ of $K_{m, 2}^{p, q} \cap \mathcal{C}_{s}^{p, q}\left(\bar{X}, E^{\otimes m}\right)$ such that $\left\{\beta_{\nu}\right\}_{\nu \geqq 1}$ converges strongly to $\theta$ in $W^{p, q}\left(X, E^{\otimes m}\right)$ (see the proof of (5.8)). This $\theta$ is the desired element. It is clear that $\|\theta\|_{m}=1$. Hence we have only to verify that $\left(\theta, \theta_{j}\right)_{m}=0$ for $1 \leqq j \leqq k$. As a sequence $\left\{\lambda_{\nu}\right\}_{\nu \geq 1}$, we may take $\left\{\lambda_{\nu}=\lambda-(\varepsilon / \nu)\right\}_{\nu \geq 1}$, where $\varepsilon$ is a positive constant such that $\lambda_{\nu}=\lambda-\varepsilon / \nu \in \Lambda_{m, \mu}$ for any $\nu$. Then the equation (5.9) can be written $Q_{m, \lambda}\left(\varphi_{\nu}, \psi\right)+(\varepsilon / \nu)\left(\varphi_{\nu}, \psi\right)_{m}=(\alpha, \psi)_{m}$ for $\psi \in W^{p, q}\left(X, E^{\otimes m}\right)$. This equation implies that each $\beta_{\nu}$ is orthogonal to the vectors $\theta_{j}$. Hence $\theta$ is orthogonal to the vectors $\theta_{j}$. Therefore $\mathrm{I}_{m, \lambda}^{s}$ has been proved.

Lastly, we prove $\mathrm{II}_{m, \lambda}^{s}$. Let $\alpha$ be an element of $\mathcal{C}_{s}^{p, q}\left(\bar{X}, E^{\otimes m}\right)$ such that $\alpha \perp K_{m, \lambda}^{p, q}$ and let $\varphi$ be the solution of the equation $\left(F_{m}-\lambda\right) \varphi=\alpha$ taken in $\mathrm{II}_{m, \lambda}^{0}$ of Theorem $\mathrm{R}_{0, \mu}$. Let $\left\{\lambda_{\nu}\right\}_{\nu \geq 1}$ be the sequence taken in the proof of $\mathrm{I}_{m, \lambda}^{s}$ and let $\left\{\varphi_{\nu}\right\}_{\nu \geqq 1}$ be the sequence of $\mathcal{C}_{s}^{p, q}\left(\bar{X}, E^{\otimes m}\right) \cap D_{F}^{p, q}$ satisfying (5.9) and (5.10). In this case, we assert the following:

$$
\left\{\left\|\varphi_{\nu}\right\|_{m}\right\}_{\nu \geq 1} \text { is bounded. }
$$

If it were unbounded, then setting $\beta_{\nu}=\varphi_{\nu} /\left\|\varphi_{\nu}\right\|_{m}$, we can construct an element $\theta$ such that $\theta \in K_{m, 2}^{p, q},\|\theta\|_{m}=1$ and $\left\|\beta_{\nu}-\theta\right\|_{m} \rightarrow 0$ as $\nu \rightarrow \infty$ (see the proof of (5.8)). On the other hand, since $\alpha \perp K_{m, \lambda}^{p, q}$, using (5.9) as in the proof of $I_{m, \lambda}^{s}$, we can verify that $\theta$ is orthogonal to the space $K_{m, \lambda}^{p, q}$. This is a contradiction. Hence (5.12) has been proved. Combining (5.10) with (5.12), we obtain that $\left\|\bar{\partial} \varphi_{\nu}\right\|_{s}$, $\left\|\bar{\partial}_{m}^{*} \varphi_{\nu}\right\|_{s}$ and $\left\|\varphi_{\nu}\right\|_{s}$ are bounded. Hence similarly to the proof of $\operatorname{II}_{m, \lambda}^{s}$ for the case $\lambda \in \Lambda_{m, \mu}$, we obtain that $\varphi$ is contained in $\mathcal{C}_{s}^{p, q}\left(\bar{X}, E^{\otimes m}\right)$ and $\left\{\varphi_{\nu}\right\},\left\{\bar{\partial} \varphi_{\nu}\right\}$ and $\left\{\bar{\partial}_{m}^{*} \varphi_{\nu}\right\}$ converge weakly to $\varphi, \bar{\partial} \varphi$ and $\bar{\partial}_{m}^{*} \varphi$ in $\mathcal{C}_{s^{*} \cdot}^{\cdot}\left(\bar{X}, E^{\otimes m}\right)$ respectively. Considering the arithmetic means of them, we obtain the desired inequality of $\mathrm{II}_{m, \lambda}^{s}$. Hence $\mathrm{II}_{m, \lambda}^{s}$ has been proved. Therefore Theorem $\mathrm{R}_{s, \mu}$ has been proved completely.

Proof of Theorem $\mathrm{N}_{s}(s \geqq 1)$. Combining Theorems $\mathrm{N}$ and $\mathrm{R}_{s, 1}$ with Proposition 5.1, ii), 2), we obtain Theorem $\mathrm{N}_{s}$, i) and ii). Since $C^{p, q-1}\left(\bar{X}, E^{\otimes m}\right)$ is dense in $L^{p, q-1}\left(X, E^{\otimes m}\right)$, combining Theorem $\mathrm{N}_{s}$, ii) with Theorem $\left.2.4,2\right)$, e), we obtain Theorem $\mathrm{N}_{s}$, iii). Hence Theorem $\mathrm{N}_{s}$ has been proved completely.

\section{§6. Application to Cohomology Theory}

Let $X$ be an $n$-dimensional complex manifold. The following definition is due to Nakano [10].

Definition 6.1. $X$ is said to be weakly 1 -complete if there exists a $C^{\infty}$ - 
plurisubharmonic function $\Phi: X \rightarrow \mathbb{R}$ such that $X_{c}:=\{x \in X \mid \Phi(x)<c\}$ is relatively compact in $X$ for any $c \in \mathbb{R}$.

$\Phi$ is called an exhaustion function. In this section, we use the notations as in Section 2. Our starting point of this section is the following representation theorem of cohomology on weakly 1-complete manifolds.

Theorem 6.2. Suppose $X$ is a weakly 1-complete manifold with exhaustion function $\Phi, E$ is a line bundle on $X$ which is positive outside a compact subset $K$ of $X$, and $F$ is a line bundle on $X$. Then for every non-critical value $c \in \mathbb{R}$ of $\Phi$ such that $c>\sup _{x \in K} \Phi(x)$, there exists a positive integer $m_{* *}$ such that the nullity $N_{L m}^{p, q}$ of the operator $L_{m}=\bar{\partial} \bar{\partial}_{m}^{*}+\bar{\partial}_{m}^{*} \bar{\partial}$ in $L^{p, q}\left(X_{c}, E^{\otimes m} \otimes F\right)$ has finite dimension and there is an isomorphism $\rho_{c}: H^{q}\left(X_{c}, \Omega^{p}\left(E^{\otimes m} \otimes F\right)\right) \rightarrow N_{L_{m}}^{p, q}$ for every $p \geqq 0, q \geqq 1$ and $m \geqq m_{* *}$.

Since this theorem can be proved by the same method used to prove Theorem 3.8 of [13], its proof is omitted here (for a detail, see [13] Chap. III).

Let $\omega: \mathscr{X} \rightarrow M$ be a regular differentiable onto map of differentiable manifolds $\mathscr{X}$ and $M$. We say that $\omega: \mathscr{X} \rightarrow M$ is a differentiable family of complex manifolds if each point of $\mathscr{X}$ has a neighborhood $U$ satisfying the condition: there exists a diffeomorphism $h$ of $U$ into $C^{n} \times \omega(U)$ such that, for each point $t \in \omega(U)$, the restriction $h_{t}$ of $h$ to $U \cap X_{t}, X_{t}=\omega^{-1}(t)$, is a biholomorphic map of $U \cap X_{t}$ into $C^{n} \times t$, where $C^{n}$ is the space of $n$-complex variables $\left(z^{1}, \cdots, z^{n}\right), n$ being the complex dimension of $X_{t}$. We call $\mathcal{E} \rightarrow \mathfrak{X} \rightarrow M$ a differentiable family over $M$ of holomorphic line bundles if $\mathcal{E} \rightarrow \mathfrak{X}$ is a differentiable complex line bundle and the restriction $E_{t} \rightarrow X_{t}$ of $\mathcal{E} \rightarrow \mathscr{X}$ to each fibre $X_{t}$ of $\mathscr{X}$ is a holomorphic line bundle over that fibre. Let $\omega: \mathfrak{X} \rightarrow M$ be a differentiable family of complex manifolds.

Definition 6.3. $\mathscr{X} \rightarrow M$ is said to be a differentiable family of weakly 1complete manifolds if there exists a $C^{\infty}$-function $\Phi: \mathscr{X} \rightarrow \mathbb{R}$ and a real number $c_{*}$ such that the restriction of $\omega$ to $\{\Phi \leqq c\}$ is proper for every $c \in \mathbb{R}$ and the restriction of $\Phi$ to each fibre $X_{t}$ of $\mathscr{X}$ is plurisubharmonic on $X_{t} \cap\left\{\Phi>c_{*}\right\}$.

$\Phi$ is called an exhaustion function and $c_{*}$ is called a pseudo-convexity bound. A differentiable family of compact complex manifolds in the sense of Kodaira [5] and a regular family of strongly pseudoconvex manifolds in the sense of Markoe and Rossi [9] are clearly differentiable families of weakly 1-complete manifolds. In both cases, the harmonic representation theorem of cohomology groups with coefficients in locally free sheaves on each fibre and the upper semicontinuity for the dimension of them hold respectively (see [2], [5], [12]). In this sense, it is natural to expect that the principle of upper semi-continuity holds for the dimension of the cohomology groups of a differentiable family of weakly 1-complete manifolds. With respect to this question, we can show the 
following theorem.

Theorem 6.4. Let $\omega: \mathfrak{X} \rightarrow M$ be a differentiable family of weakly 1-complete manifolds with exhaustion function $\Phi$ and pseudoconvexity bound $c_{*}$. Let $\mathcal{E} \rightarrow \mathfrak{X}$ $\rightarrow M$ and $\mathscr{I} \rightarrow \mathfrak{X} \rightarrow M$ be differentiable families over $M$ of holomorphic line bundles. $E_{t} \rightarrow X_{t}$ and $F_{t} \rightarrow X_{t}$ denote the restriction of $\mathcal{E} \rightarrow \mathfrak{X}$ and $\mathscr{I} \rightarrow \mathfrak{X}$ to each fibre $X_{t}$ of $\mathscr{X}$ respectively.

Assumption: There exist a closed subset $\mathcal{K}$ of $\mathscr{X}$ and a fibre metric a of $\mathcal{E} \rightarrow \mathfrak{X}$ such that the restriction of $\omega$ to $\mathcal{K}$ is proper and the restriction $\boldsymbol{a}_{t}$ of $\boldsymbol{a}$ to each line bundle $E_{t} \rightarrow X_{t}$ gives the positivity of its line bundle on $X_{t} \backslash K_{t}$, where $K_{t}=\mathcal{K} \cap X_{t}$ for $t \in M$.

Conclusion: For any point $t_{0} \in M$ and non-critical value $c>\max \left\{c_{*}, \sup _{x \in K_{t_{0}}} \Phi(x)\right\}$ of $\Phi$, there exist an open neighborhood $V$ of $t_{0}$ in $M$ and a positive integer $m(\mathscr{T}, V, c)$ such that if $p \geqq 0, q \geqq 1, t \in V$ and $m \geqq m(\mathscr{F}, V, c)$, then $\operatorname{dim}_{C} H^{q}\left(X_{t, c}, \Omega^{p}\left(E_{t}^{\otimes m} \otimes F_{t}\right)\right)$ $<\infty$ and $\operatorname{dim}_{C} H^{q}\left(X_{t, c}, \Omega^{p}\left(E_{t}^{\otimes m} \otimes F_{t}\right)\right) \leqq \operatorname{dim}_{c} H^{q}\left(X_{t_{0}, c}, \Omega^{p}\left(E_{t_{0}}^{\otimes m} \otimes F_{t_{0}}\right)\right)$, where $X_{t, c}=$ $X_{t} \cap\{\Phi<c\}$ for $t \in M$.

Proof. We give only the proof of the case $\mathscr{T} \rightarrow \mathfrak{X} \rightarrow M$ is a differentiable family over $M$ of analytically trivial line bundles. In view of Remark 3.3, the proof of the another case is quite similar. Taking a relatively compact neighborhood $W$ of $t_{0}$ in $M$ and a closed subset $\mathcal{K}^{\prime}$ of $\mathscr{X}$ such that $\mathcal{K} \subseteq \mathcal{K}^{\prime}$ and $c>$ $\sup \left\{\Phi(x) \mid x \in \mathcal{K}^{\prime} \cap \omega^{-1}(\bar{W})\right\}$, we can construct a differentiable family of hermitian metrics $\left\{d s_{t}^{2}\right\}_{t \in W}$ such that $d s_{t}^{2}=\sum g_{i, \alpha \bar{\beta}}\left(z_{i}, t\right) d z_{i}^{\alpha} d z_{i}^{\beta}$ is a hermitian metric on $X_{t}$ which is Kähler on $X_{t} \backslash K_{t}^{\prime}, K_{t}^{\prime}=\kappa^{\prime} \cap X_{t}$, and the functions $g_{i, \alpha \bar{\beta}}\left(z_{i}, t\right)$ are differentiable ones of $z_{i}$ and $t$. With respect to the metrics $d s_{t}^{2}$ and $\boldsymbol{a}_{t}$, we define the notations as in Section 2. For each $t \in W$, the constants appeared in the calculations of Section 4 depend on the functions $\left\{g_{i, \alpha}\left(z_{i}, t\right)\right\}, \boldsymbol{a}_{t}=\left\{a_{i}\left(z_{i}, t\right)\right\}$ and their derivatives with respect to the fibre coordinates $\left(z_{i}^{\alpha}\right)$. Hence they depend continuously on $t \in W$. Taking an open neighborhood $V \Subset W$ of $t_{0}$, we may assume that those constants are independent of $t \in V$. Therefore we can take the positive integers taken in the assertions of Propositions 4.4, 4.5 and Theorem 4.8 uniformly with respect to $t \in V$.

By definition, each fibre $X_{t}$ is a weakly 1-complete manifold. Hence, on each fibre $X_{t}$, Theorem 6.2 holds. Moreover the uniformity of the estimate of Proposition 4.4 implies that the integer $m_{*}$ determined in Theorem 6.2 can be taken uniformly with respect to $t$ i. e. there exists a positive integer $m_{*}$ not depending on $t$ such that the space $N_{L_{m}, t}^{p, q} \subseteq L^{p, q}\left(X_{t, c}, E_{t}^{\otimes m}\right)$, where $L_{m, t}=\bar{\partial}_{t} \bar{\partial}_{m, t}^{*}$ $+\bar{\partial}_{m, t}^{*} \bar{\partial}_{t}$, is finite dimensional and the cohomology group $H^{q}\left(X_{t, c}, \Omega^{p}\left(E_{t}^{\otimes m}\right)\right)$ is isomorphic to $N_{L_{m}, t}^{p, q}$ for any $p \geqq 0, q \geqq 1, t \in V$ and $m \geqq m_{*}$. Here we apply Theorem $\mathrm{N}_{s}$ to our situation. For $s=1$, we can take the integer $m(1) \geqq m_{*}$ determined in Theorem $\mathrm{N}_{1}$ uniformly with respect to $t$ and fix an integer $m \geqq m(1)$. The former assertion of the theorem follows from the above representation theorem. The 
latter one is shown as follows. We may put $t_{0}=0$. Let $d_{0}=\operatorname{dim}_{C} N_{L_{m}, 0_{0}}^{p . q}$ If the upper semi-continuity did not hold, then there would be a sequence $\left\{t_{\nu}\right\}_{\nu \geq 1}$ of points in $V$ such that $t_{\nu} \rightarrow 0$ as $\nu \rightarrow \infty$ and $\operatorname{dim}_{C} N_{L_{m}, t_{\nu}}^{p, q}>d_{0}$. By Theorem $N_{1}$, there exist vectors $\left\{\theta_{\nu, l}\right\}_{1 \leqq l \leqq d_{0^{+1}}}$ of $N_{L_{m}, t_{\nu}}^{p, q} \cap \mathcal{C}_{1}^{p, q}\left(\bar{X}_{t_{\nu}, c}, E_{t_{\nu}}^{\otimes m}\right)$ such that $\left(\theta_{\nu, k}, \theta_{\nu, l}\right\}_{m, t_{\nu}}=$ $\delta_{k l}$. Then $\left\{\left\|\theta_{\nu, l}\right\|_{1, t_{\nu}}\right\}$ is bounded from the way of constructing the vectors $\theta_{\nu, l}$ (see the proof of Theorem $\mathrm{R}_{s, \mu}$ ). On the other hand, we can assume that there exists a diffeomorphism $\Psi: \omega^{-1}(V) \cap\left\{\Phi<c^{\prime}\right\} \rightarrow X_{0, c^{\prime}} \times V$ satisfying $\omega=\pi \circ \Psi$ where $c^{\prime}$ is a non-critical value of $\Phi$ with $c^{\prime}>c$ and $\pi$ is the projection to the second factor. Then the restriction $\Psi_{t}$ of $\Psi$ to each fibre $X_{t, c^{\prime}}$ yields a diffeomorphism of $X_{t, c^{\prime}}$ to $X_{0, c^{\prime}}$. We set $\theta_{\nu, l}^{*}=\left(\Psi_{t_{\nu}}^{-1}\right)^{*} \theta_{\nu, l}$ for $\nu \geqq 1$ and $1 \leqq l \leqq d_{0}+1$. Then, by the local invariance of the Sobolev spaces under coordinate transformations, each $\theta_{\nu, l}^{*}$ is an element of $\bigoplus_{s+t=r} \mathcal{C}_{1}^{s, t}\left(\bar{X}_{0, c}, E_{0}^{\otimes m}\right)(r=p+q)$ and $\left\{\left\|\theta_{\nu, l}^{*}\right\|_{1,0}\right\}$ is bounded in $\bigoplus_{s+t=r} \mathcal{C}_{1}^{s, t}\left(\bar{X}_{0, c}, E_{0}^{\otimes m}\right)$ since $\left\{\left\|\theta_{\nu, l}\right\|_{1, t_{\nu}}\right\}$ is bounded. Since the complex structure on $X_{t, c^{\prime}}$ depends differentiably on $t$, by Lemma $\left.2.1,1\right)$, there exist vectors $\left\{\theta_{l}\right\}_{1 \leqq l \leqq d_{0}+1}$ of $L^{p, q}\left(X_{0, c} E_{0}^{\otimes m}\right)$ such that $\left\|\theta_{\nu, l}^{*}-\theta_{l}\right\|_{m, 0} \rightarrow 0$ as $\nu \rightarrow \infty$ for any $l$. Then we have $\left(\theta_{k}, \theta_{l}\right)_{m, 0}=\delta_{k l}$ by continuity. Moreover for $\varphi \in C_{c}^{p, q+1}\left(X_{0, c}, E_{0}^{\otimes m}\right)$ and $\phi \in$ $C^{p, q-1}\left(\bar{X}_{0, c}, E_{0}^{\otimes m}\right)$, we have

$$
\begin{aligned}
\left(\theta_{l}, \vartheta_{m, 0} \varphi\right)_{m, 0}= & \lim _{\nu \rightarrow \infty}\left(\theta_{\nu, l}^{*}, \vartheta_{m, 0} \varphi\right)_{m, 0} \\
= & \lim _{\nu \rightarrow \infty}\left[\left(\theta_{\nu, l}, \vartheta_{m, t_{\nu}}\left(\Psi_{t_{\nu}}\right)^{*} \varphi\right)_{m, t_{\nu}}\right. \\
& +\left(\theta_{\nu, l},\left(\left(\Psi_{t_{\nu}}\right) * \vartheta_{m, 0}-\vartheta_{m, t_{\nu}}\left(\Psi_{t_{\nu}}\right) *\right)_{m, t_{\nu}}\right] \\
= & 0
\end{aligned}
$$

and

$$
\begin{aligned}
\left(\theta_{l}, \bar{\partial}_{0} \psi\right)_{m, 0}= & \lim _{\nu \rightarrow \infty}\left(\theta_{\nu, l}^{*}, \bar{\partial}_{0} \psi\right)_{m, 0} \\
= & \lim _{\nu \rightarrow \infty}\left[\left(\theta_{\nu, l}, \bar{\partial}_{t_{\nu}}\left(\Psi_{t_{\nu}}\right)^{*} \psi\right)_{m, t_{\nu}}\right. \\
& \left.+\left(\theta_{\nu, l},\left(\left(\Psi_{t_{\nu}}\right) * \bar{\partial}_{0}-\bar{\partial}_{t_{\nu}}\left(\Psi_{t_{\nu}}\right)^{*}\right) \psi\right)_{m, t_{\nu}}\right] \\
= & 0
\end{aligned}
$$

for any $l$ respectively. Hence we have $\bar{\partial}_{0} \theta_{l}=\bar{\partial}_{m, 0}^{*} \theta_{l}=0$ for any $l$. Therefore $d_{0}=\operatorname{dim}_{C} N_{L m, 0}^{p, q} \geqq d_{0}+1$. This is a contradiction. Hence the theorem has been proved completely.

\section{§ 7. Appendix}

I. Let the notations be as in Sections 2 and 4 . Let $L=\left(L_{1}, L_{2}, \cdots, L_{q}\right)$ : $\stackrel{q}{\oplus}\left[C^{0,0}\left(\bar{X}, E^{\otimes m}\right)\right] \rightarrow C^{0,0}\left(\bar{X}, E^{\otimes m}\right)$ be a differential operator of order one defined as follows :

$$
\begin{array}{r}
\text { For any } u={ }^{t}\left(u_{1}, u_{2}, \cdots, u_{q}\right) \in \stackrel{q}{\oplus}\left[C^{0,0}\left(\bar{X}, E^{\otimes m}\right)\right] \\
L u=\sum_{\alpha=1}^{q} L_{\alpha} u_{\alpha}
\end{array}
$$


and

$$
\left(L_{\alpha} u_{\alpha}\right)_{i}=\left(\sum_{k=1}^{2 n} C_{i, a, k}^{(1)} D_{i}^{k}+C_{i, \alpha, m}^{(2)}\right) u_{\alpha, i} \quad(1 \leqq \alpha \leqq q)
$$

on every $U_{i} \cap \bar{X}$,

where $D_{\imath}^{k}=\sqrt{-1}\left(\partial / \partial x_{\imath}^{k}\right)\left(\left(x_{i}^{1}, \cdots, x_{\imath}^{2 n}\right)\right.$ are real local coordinates on $\left.U_{i}\right)$ and $C_{i, \alpha, k}^{(1)}$, $C_{i, \alpha, m}^{(2)}$ are $C^{\infty}$-functions on $U_{i}$ such that $C_{i, a, k}^{(1)}$ does not depend on $m$ but $C_{i, \alpha, m}^{(2)}$ may depend on $m$.

Lemma 4.3 is derived from the following lemma.

Lemma A.1.1. For any $i \in\{1, \cdots, N\}, m \geqq 1, s \geqq 0$ and real-valued $C^{\infty}$-functions $\rho, \chi, \eta$ in $C_{c}^{0,0}\left(U_{i}\right)$ such that $\chi \equiv 1$ on $\operatorname{supp} \rho$ and $\eta \equiv 1$ on $\operatorname{supp} \chi$, there exist positive constants $C_{s}$ and $C_{m, s}^{(\gamma)}(\gamma=1,2)$ such that

1) for any multi-index $\sigma=\left(\sigma_{1}, \cdots, \sigma_{2 n}\right)$ such that $|\sigma|=s$ if $U_{i} \in U_{1},|\sigma|=s$ and $\sigma_{2 n}=0$ if $U_{i} \in \mathcal{U}_{2}$, and $u \in \stackrel{q}{\oplus}\left[C^{0,0}\left(\bar{X}, E^{\otimes m}\right)\right]$,

$$
\begin{aligned}
\left\|L \Delta_{l, \rho}^{\sigma} u\right\|_{m}^{2} \leqq & C_{s} \sum_{\substack{1, \sum \leq s \\
1 \leq \alpha \leq q}}\left\|\Delta_{l, \chi}^{\theta} u_{a}\right\|_{m}^{2}+2 \operatorname{Re}\left(L u, L \Delta_{l, \rho, m}^{\sigma *} \Delta_{l, \rho}^{\sigma} u\right)_{m} \\
& +C_{m, s}^{(1)} \sum_{\substack{\theta|\theta| \leq s-1 \\
1 \leqq \alpha \leq q}}\left\|\Delta_{l, \eta}^{\theta} u_{\alpha}\right\|_{m}^{2}+C_{m, s}^{(2)} \sum_{|\theta| \leq s-1} \operatorname{Re}\left(L u, L \Delta_{l, x_{,}}^{\theta *} \Delta_{l, \chi}^{\theta} u\right)_{m}
\end{aligned}
$$

where $\Delta_{l, \rho}^{\sigma} u={ }^{t}\left(\Delta_{l, \rho}^{\sigma} u_{1}, \cdots, \Delta_{l, \rho}^{\sigma} u_{q}\right)$ and so on

2) $C_{s}$ (resp. $\left.C_{m, s}^{(r)}\right)$ depends on $s$ (resp. $m$ and $s$ ) and $C_{m, 0}^{(r)}=0(\gamma=1,2)$.

Proof. We set

Then we have

$$
\begin{gathered}
\Lambda(u)=\left(\left[L, \Delta_{l, \rho}^{\sigma}\right] u, L \Delta_{l, \rho}^{\sigma} u\right)_{m}-\left(\left[L, \Delta_{l, \rho}^{\sigma *}\right] \Delta_{l, \rho}^{\sigma} u, L u\right)_{m} \\
\text { for } u \in \stackrel{q}{\oplus}\left[C^{0,0}\left(\bar{X}, E^{\otimes m}\right)\right] .
\end{gathered}
$$

$$
\Lambda(u)+\overline{\Lambda(u)}=2\left\{\left\|L \Delta_{l, \rho}^{\sigma} u\right\|_{m}^{2}-\operatorname{Re}\left(L u, L \Delta_{l, \rho, m}^{\sigma *} \Delta_{l, \rho}^{\sigma} u\right)_{m}\right\} .
$$

Since the supports of integrands are compact in $U_{i}$ and we have only to prove the required estimate only on $U_{i}$, we may consider that $(,)_{m}$ is an inner product on $C^{0,0} \overline{\left(X \cap U_{i}\right)}$ with a weight $a_{i}^{m}$ i.e. $(,)_{m}=\left(, a_{i}^{m}\right)$ on $C^{0,0} \overline{\left(X \cap U_{i}\right)}$. Hence $\Delta_{l, \rho, m}^{\sigma *}$ can be written in the following way:

$$
\Delta_{l, \rho, m}^{\sigma *} v=\Delta_{l, \rho}^{\sigma} v+\Delta_{m} v
$$

and

$$
\begin{aligned}
\Delta_{m} v & =\sum_{|\theta| \leq s-1} b_{m, \theta} \Delta_{l, \chi}^{\theta} v \\
& \text { for every } v \in C^{0,0} \overline{\left(X \cap U_{i}\right)}
\end{aligned}
$$

where $b_{m, \theta}$ are $C^{\infty}$-functions on $U_{i}$ whose supports are contained in the support of $\rho$ and depend on $m$.

Moreover we recall the following fact.

(A.1.5) If $D_{1}$ and $D_{2}$ are differential operators of order $s_{1}$ and $s_{2}$ respectively, then $\left[D_{1}, D_{2}\right]$ is a differential operator of order $s_{1}+s_{2}-1$. 
We rewrite $\Lambda(u)$ as follows:

$$
\begin{aligned}
\Lambda(u)= & \left(\left[L, \Delta_{l, \rho}^{\sigma}\right] u, L \Delta_{l, \rho}^{\sigma} u\right)_{m}+\left(\left[L, \Delta_{l, \rho, m}^{\sigma *}\right] u,\left[L, \Delta_{l, \rho, m}^{\sigma *}\right] u\right)_{m} \\
& +\left(\left[\left[L, \Delta_{l, \rho, m}^{\sigma *}\right], \Delta_{l, \rho}^{\sigma}\right] u, L u\right)_{m}+\left(\left[L, \Delta_{l, \rho, m}^{\sigma *}\right] u, L \Delta_{l, \rho, m}^{\sigma *} u\right)_{m} .
\end{aligned}
$$

Since $\left[L, \Delta_{l, \rho}^{\sigma}\right]$ and $\left[L, \Delta_{l, \rho, m}^{\sigma *}\right]$ are differential operators of order $s$ by (A.1.5) and the coefficients of the highest order terms of them do not depend on $m$, we have

$$
\begin{aligned}
& \left|\left(\left[L, \Delta_{l, \rho}^{\sigma}\right] u, L \Delta_{l, \rho}^{\sigma} u\right)_{m}\right| \leqq(*)+\frac{1}{2}\left\|L \Delta_{l, \rho}^{\sigma} u\right\|_{m}^{2} \\
& \quad\left\|\left[L, \Delta_{l, \rho, m}^{\sigma *}\right] u\right\|_{m}^{2} \leqq(*) \\
& \left|\left(\left[L, \Delta_{l, \rho, m}^{\sigma *}\right] u, L \Delta_{l, \rho, m}^{\sigma *} u\right)_{m}\right| \leqq(*)+\frac{1}{2}\left\|L \Delta_{l, \rho}^{\sigma} u\right\|_{m}^{2}+\left\|L \Delta_{m} u\right\|_{m}^{2}
\end{aligned}
$$

where $\quad(*):=C_{s} \sum_{\substack{1 \theta 1=s \\ 1 \leqq \alpha \leqq q}}\left\|\Delta_{l, \chi}^{\sigma} u_{a}\right\|_{m}^{2}+C_{m, s}^{\prime} \sum_{\substack{1,1 \leq \leq-1 \\ 1 \leq a \leq q}}\left\|\Delta_{l, \chi}^{\theta} u_{\alpha}\right\|_{m}^{2}$

and $C_{s}$ (resp. $C_{m, s}^{\prime}$ ) is a positive constant depending on $s$ (resp. $m$ and $s$ ).

By (A.1.5), $\left[\left[L, \Delta_{l, \rho, m}^{\sigma *}\right], \Delta_{l, \rho}^{\sigma}\right]$ is a differential operator of order $2 s-1$ and the coefficients of the highest order terms of this operator do not depend on $m$. Hence by integration by parts, we have

$$
\left|\left(\left[\left[L, \Delta_{l, \rho, m}^{\sigma *}\right], \Delta_{l, \rho}^{\sigma}\right] u, L u\right)_{m}\right| \leqq(*) .
$$

From (A.1.3), (A.1.6), (A.1.7) and (A.1.8), we have

$$
\left\|L \Delta_{l, \rho}^{\sigma} u\right\|_{m}^{2} \leqq(*)+2 \operatorname{Re}\left(L u, L \Delta_{l, \rho, m}^{\sigma *} \Delta_{l, \rho}^{\sigma} u\right)_{m}+\left\|L \Delta_{m} u\right\|_{m}^{2} .
$$

From (A.1.4), there exists a positive constant $C_{m, s}^{\prime \prime}$ depending on $m$ and $s$ such that

$$
\left\|L \Delta_{m} u\right\|_{m}^{2} \leqq C_{m, s}^{\prime \prime}\left\{\sum_{|\theta| \leqq s-1}\left\|L \Delta_{l, \chi}^{\theta} u\right\|_{m}^{2}+\sum_{\substack{1 \theta_{1 \leq s-1} \leq \sum_{1 \leq \alpha} \\ 1 \leqq \alpha \leq \alpha}}\left\|\Delta_{l, \chi}^{\theta} u_{\alpha}\right\|_{m}^{2}\right\} .
$$

Applying the above argument to $\left\|L \Delta_{l, \chi}^{\theta} u\right\|_{m}^{2}$ of (A.1.10), there exists a positive constant $C_{m, s-1}$ depending on $m$ and $s-1$ such that for any multi-index $\theta=$ $\left(\theta_{1}, \cdots, \theta_{2 n}\right)$ so that $|\theta| \leqq s-1$ or $|\theta| \leqq s-1$ and $\theta_{2 n}=0$

$$
\left\|L \Delta_{l, \chi}^{\theta} u\right\|_{m}^{2} \leqq C_{m, s-1} \sum_{\substack{|\gamma| \leq s-1 \\ 1 \leqq \alpha \leq q}}\left\|\Delta_{l, \eta}^{\gamma} u_{a}\right\|_{m}^{2}+2 \operatorname{Re}\left(L u, L \Delta_{l, \chi, m}^{\theta *} \Delta_{l, \chi}^{\theta} u\right)_{m} .
$$

From (A.1.9), (A.1.10) and (A.1.11), we obtain (A.1.2).

q. e. d.

II. Let $X$ be a relatively compact domain with smooth boundary $\partial X$ on an $n$ dimensional complex manifold $M$ and let $E$ be a holomorphic line bundle on $M$. For a suitable covering $\left\{U_{i}\right\}_{i \in I}$ of $M$, we fix a hermitian metric $a=\left\{a_{i}\right\}$ of $E$ and a hermitian metric $d s^{2}=\sum_{\alpha, \beta=1}^{n} g_{i, \alpha \bar{\beta}} d z_{i}^{\alpha} d z_{\imath}^{\bar{\beta}}$ on $M$ such that $d s^{2}$ is Kähler on a neighborhood $U^{*}$ of $\partial X$. Let $\bar{\nabla}$ be the covariant differentiation associated to $d s^{2}$. With respect to these metrics, we define the notations as in Section 2. By a 
complex tensor calculus for Kähler manifolds with boundary, we obtain the following theorem (see [13] Chap. I, 1.1).

Theorem A.2.1. If $m \geqq 1$, then

$$
\begin{aligned}
& \|\bar{\partial} \varphi\|_{m}^{2}+\left\|\bar{\partial}_{m}^{*} \varphi\right\|_{m}^{2} \\
& =\|\bar{\nabla} \varphi\|_{m}^{2}+\int_{X} a_{i}{ }^{m} \sum q\left(\delta^{\sigma}{ }_{\tau}\left[m \Theta \frac{\bar{\beta}}{\alpha}+R \frac{\bar{\beta}}{\alpha}\right]-p R^{\sigma}{ }_{\tau \bar{\alpha}}{ }^{\bar{\beta}}\right) \\
& \times \varphi_{i, \sigma C_{p-1}, \bar{\beta} \bar{D}_{q-1}} \overline{\varphi_{i}{ }^{\bar{\tau} \bar{C}_{p-1}, \alpha D_{q-1}}} d V \\
& +\int_{\partial X} a_{i}{ }^{m}|\operatorname{grad} h|_{d^{2} s^{2}}^{-2} \sum \frac{\partial^{2} h}{\partial z_{i}^{\alpha} \partial \bar{z}_{\imath}^{\beta}} \varphi_{i, C_{p} \bar{D}_{q-1}} \overline{\varphi_{i}^{\bar{C}_{p}, \beta D_{q-1}}} d S
\end{aligned}
$$

for any $\varphi \in B^{p, q}\left(\bar{X}, E^{\otimes m}\right)$ such that $\operatorname{supp} \varphi \Subset U^{*}, p \geqq 0$ and $q \geqq 1$, where $\|\bar{\nabla} \varphi\|_{m}^{2}=$ $\int_{X} \sum g_{i}^{\bar{\beta} \alpha} \bar{\nabla}_{\beta} \varphi_{i, C_{p}, \bar{D}_{q}} \overline{\bar{\nabla}_{\alpha} \varphi_{i}^{\bar{C}_{p}, D_{q}}} d V$, $R_{\beta \bar{\nu} \lambda}^{\alpha}=-\frac{\partial}{\partial \bar{z}_{i}^{\nu}}\left(\sum g_{i}^{\bar{\sigma} \alpha} \frac{\partial}{\partial z_{i}^{\lambda}}\left(g_{i, \beta \bar{\sigma}}\right)\right)$ is the Riemann curvature tensor, $R_{\lambda \bar{\nu}}=-\frac{\partial^{2}}{\partial \bar{z}_{i}^{\nu} \partial z_{i}^{\lambda}}\left(\log \operatorname{det}\left(g_{i, \alpha \bar{\beta}}\right)\right)$ is the Ricci curvature tensor, $\Theta_{\lambda \nu}=-\frac{\partial^{2}}{\partial z_{\imath}^{\lambda} \partial \bar{z}_{i}^{\nu}}\left(\log a_{i}\right)$ is the curvature tensor of $E$ and $\delta^{\sigma}{ }_{\tau}$ denotes the Kronecker's delta.

We prove Proposition 4.4 using this theorem.

Proof of Proposition 4.4. We set ourselves in the situation of Lemma 4.1. Let $\chi$ be a $C^{\infty}$-function on $M$ such that supp $\chi \in \Omega^{\prime}$ and $\chi \equiv 1$ on $\bar{\Omega}$. Then we can apply the formula (A.2.2) to $\chi_{\varphi}$. Since the third term of the right-hand side of (A.2.2) is non-negative by the pseudoconvexity of $\partial X$, we obtain

$$
\begin{aligned}
& \left\|\bar{\nabla}\left(\chi_{\varphi}\right)\right\|_{m}^{2}+\int_{X} a_{i}{ }^{m} \sum q\left(\delta^{\sigma}{ }_{\tau}\left[m \Theta_{\frac{\bar{\beta}}{\alpha}}^{\bar{\beta}}+R_{\frac{\bar{\beta}}{\alpha}}^{\bar{\beta}}\right]-p R^{\sigma}{ }_{\tau \bar{\alpha}}{ }^{\bar{\beta}}\right) \\
& \times\left(\chi_{\varphi}\right)_{i, \sigma C_{p-1}, \bar{\beta} \bar{D}_{q-1}} \overline{\left(\chi_{\varphi}\right)_{i}^{\bar{\tau} \bar{C}} p-1, \alpha D_{q-1}} d V \\
& \leqq\left\|\bar{\partial}\left(\chi_{\varphi}\right)\right\|_{m}^{2}+\left\|\bar{\partial}_{m}^{*}\left(\chi_{\varphi}\right)\right\|_{m}^{2} \text {. }
\end{aligned}
$$

Since the integrand of the first term of the left-hand side of (A.2.3) is nonnegative on $\Omega^{\prime}$, we have

$$
\|\bar{\nabla} \varphi\|_{m, X \backslash K}^{2} \leqq\left\|\bar{\nabla}\left(\chi_{\varphi}\right)\right\|_{m}^{2} \quad \text { where } K=X \backslash(X \cap \Omega) .
$$

From the construction of $d s^{2}$, the matrix $\left(g_{i, \alpha \bar{\beta}}\right)$ coincides with the one $\left(\Theta_{\alpha \bar{\beta}}\right)$ at each point of $\Omega^{\prime}$. Hence we have $\Theta_{\bar{\alpha}}^{\bar{\beta}}=\sum_{\gamma=1}^{n} g_{i}{ }^{\bar{\beta} r} \Theta_{\gamma \bar{\alpha}}=\delta^{\beta}{ }_{\alpha}$. On the other hand, there exists a positive constant $C$ not depending on $m$ such that the hermitian form $\sum q\left(\delta^{\sigma}{ }_{\tau} R_{\frac{\beta}{\alpha}}^{\bar{\beta}}-p R^{\sigma}{ }_{\tau} \bar{a} \bar{\beta}\right)\left(\chi_{\varphi}\right)_{i, \sigma C} C_{p-1}, \bar{\beta} \bar{D}_{q-1} \overline{\left.\chi_{\varphi}\right)_{i}{ }_{i} \overline{\bar{C}} \bar{C}_{p-1}, \alpha D_{q-1}}$ is greater than

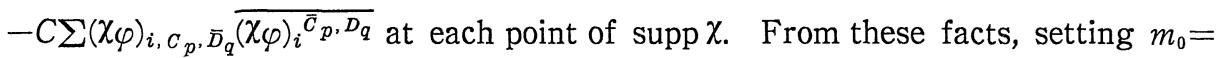


$[C]+1$, for every $m \geqq m_{0}$, we have

$$
\begin{aligned}
\left(m-m_{0}\right)\|\varphi\|_{m, X \backslash K}^{2} & \leqq\left(m-m_{0}\right)\|\chi \varphi\|_{m}^{2} \\
& \leqq \text { the second term of the left-hand side of (A.2.3). }
\end{aligned}
$$

Moreover we have

$$
\begin{aligned}
\|\bar{\partial}(\chi \varphi)\|_{m}^{2}+\left\|\bar{\partial}_{m}^{*}(\chi \varphi)\right\|_{m}^{2} & \leqq 2\left\{\|\bar{\partial} \chi \wedge \varphi\|_{m}^{2}+\|\partial \chi \wedge * \varphi\|_{m}^{2}+\|\chi \bar{\partial} \varphi\|_{m}^{2}+\left\|\chi \bar{\partial}_{m}^{*} \varphi\right\|_{m}^{2}\right\} \\
& \leqq C\left\{\|\bar{\partial} \varphi\|_{m}^{2}+\left\|\bar{\partial}_{m}^{*} \varphi\right\|_{m}^{2}+\|\varphi\|_{m, X \backslash K}^{2}\right\}
\end{aligned}
$$

for a positive constant $C \geqq 4 \cdot \max \left\{1, c_{0} \cdot \sup |\operatorname{grad} \chi|_{d_{s 2}}(x)\right\}$ and $m \geqq 1$ where $c_{0}$ is a positive constant depending only on the dimension of $M$.

From (A.2.4), (A.2.5) and (A.2.6), we obtain the desired estimate. q.e.d.

III. Let $\left(H,(,)_{H}\right)$ be a Hilbert space over the complex field $C$ and let $T: H$ $\rightarrow H$ be a self-adjoint operator i. e. $T$ is densely defined and $T=T^{*}$. Let $\sigma(T)$ be the spectrum of $T$. Then since $T$ is self-adjoint, $\sigma(T)$ is decomposed into the essential spectrum $\sigma_{e}(T)$ and the discrete spectrum $\sigma_{d}(T)$, where $\sigma_{e}(T)$ is the points set of $\sigma(T)$ that are either accumulation points of $\sigma(T)$ or isolated eigenvalues of infinite multiplicity and $\sigma_{d}(T)$ is the set of isolated eigenvalues of finite multiplicity. One of the characterization of $\sigma_{e}(T)$ is given by the following lemma (see [15] Theorem 7.24).

Lemma A.3.1. A real number $\lambda$ is contained in $\sigma_{e}(T)$ if and only if there exists a sequence $\left\{f_{\nu}\right\}_{\nu \geqq 1}$ of $D_{T}$ such that $\left\{f_{\nu}\right\}_{\nu \geqq 1}$ converges weakly to zero, $\lim _{\nu \rightarrow \infty} \inf \left\|f_{\nu}\right\|_{H}>0$ and $\left\{(T-\lambda) f_{\nu}\right\}_{\nu \geq 1}$ converges strongly to zero.

Using this lemma, we can easily prove the following theorem. For the simplicity of its proof, the detail is left to the reader.

Theorem A.3.2. Let $H$ and $T$ be as above and let $\lambda$ be an eigenvalue of $T$ of finite multiplicity i.e. $0<\operatorname{dim}_{C} N_{T-\lambda}<\infty$. Then the following two conditions are equivalent

a) $\lambda \in \sigma_{d}(T)$

b) there exists a positive constant $C$ such that $\|f\|_{H} \leqq C\|(T-\lambda) f\|_{H}$ if $f \in D_{T}$ and $f \perp N_{T-\lambda}$.

\section{References}

[1] Catlin, D., Boundary behavior of holomorphic functions on pseudoconvex domains, J. Diff. Geom., 15 (1980), 605-625.

[2] Folland, G.B. and Kohn, J.J., The Neumann problem for the Cauchy-Riemann complex, Ann. of Math. Studies, 75, P.U. Press, 1975.

[3] Grauert, H., Bemerkenswerte pseudokonvexe Mannigfaltigkeiten, Math. Z., 81 (1963), 377-391. 
[4] Hörmander, L., $L^{2}$-estimates and existence theorems for the $\bar{\sigma}$-operator, Acta Math., 113 (1965), 89-152.

[5] Kodaira, K. and Spencer, D.C., On deformations of complex analytic structures, III, Stability theorems for complex structures, Ann. of Math., 71 (1960), 43-76.

[6] Kohn, J. J., Global regularity for $\bar{\partial}$ on weakly pseudoconvex manifolds, Trans. Amer. Math. Soc., 181 (1973), 273-292.

[7] , Propagation of singularities for the Cauchy-Riemann equations, C.I.M.E. Conf. Complex Analysis, (1973), 179-280.

[8] Kohn, J. J. and Nirenberg, L., Non-coercive boundary value problems, Comm. Pure Appl. Math., 18 (1965), 443-492.

[9] Markoe, A. and Rossi, H., Families of strongly pseudoconvex manifolds, Symp. on several complex variables, Park City, Utah., Lecture Notes in Math., 184, Springer, 1970, 182-207.

[10] Nakano, S., Vanishing theorems for weakly 1-complete manifolds, Number theory, algebraic geometry and commutative algebra, in honor of $Y$. Akizuki, Kinokuniya, 1973, 169-179.

[11] Ohsawa, T., Isomorphism theorems for cohomology groups of weakly 1-complete manifolds, Publ. RIMS, Kyoto Univ., 18 (1982), 191-232.

[12] Siu, Y.T., Dimension of sheaf cohomology groups under holomorphic deformation, Math. Ann., 192 (1971), 203-215.

[13] Takegoshi, K., Representation theorems of cohomology on weakly 1-complete manifolds, Publ. RIMS, Kyoto Univ., 18 (1982), 551-606.

[14] — - On weakly 1-complete surfaces without non-constant holomorphic tions, Publ. RIMS, Kyoto Univ., 18 (1982), 1175-1183.

[15] Weidmann, J., Linear operators in Hilbert spaces, Springer Verlag. 